\title{
Trajectory Design and Maintenance of the Martian Moons eXploration Mission Around Phobos
}

\author{
Nicola Baresi* \\ University of Surrey, Surrey, England GU2 7XH, United Kingdom \\ and \\ Diogene A. Dei Tos,, \pm Hitoshi Ikeda,, and Yasuhiro Kawakatsu $\$$ \\ Japan Aerospace Exploration Agency, Sagamihara 252-0222, Japan \\ https://doi.org/10.2514/1.G005041
}

\begin{abstract}
The Martian Moons eXploration mission, currently under development by the Japan Aerospace Exploration Agency (JAXA), will be launched in 2024 with the goal of retrieving pristine samples from the surface of Phobos. Soon after arrival, the spacecraft will inject into retrograde relative trajectories known as quasi-satellite orbits and study the geophysical environment of the Martian moon for more than three years. This paper presents the orbit design and maintenance strategy of the Martian Moons eXploration mission in the framework of the elliptic Hill problem with ellipsoidal secondary. This paper first introduces a numerical continuation procedure on the eccentricity of Phobos to replace purely periodic solutions with families of quasi-periodic invariant tori. Two-dimensional torus maps can be then constructed and used to represent physical quantities of interest, as well as to generate reference trajectories at arbitrary epochs. Sensitivity and stability analyses are carried out to investigate the dynamic properties of retrograde relative trajectories in the elliptic case. Finally, a linear quadratic regulator is implemented in order to assess the robustness of the computed trajectories under injection, navigation, and execution errors. Monte Carlo simulations demonstrate that the baseline quasi-satellite orbits of the Martian Moons eXploration mission can be maintained with as low as $6.265 \mathrm{~m} / \mathrm{s}$ per month.
\end{abstract}

\section{Nomenclature}

$a_{P}, e_{P}, p_{P}=$ Phobos semimajor axis $(\mathrm{km})$, eccentricity, and semiparameter semimajor axis $(\mathrm{km})$

$B \quad=$ control input matrix

$C=$ Cauchy-Green tensor

$\boldsymbol{c}_{k}, \boldsymbol{d}_{k} \quad=\quad$ vectors of Fourier coefficients

$\boldsymbol{e}$

$=$ error vector between spacecraft reference and estimated states in normalized pulsating coordinates

$\mathcal{F} \quad=\quad$ reduced error vector

$\boldsymbol{F} \quad=\quad$ error vector of Newton's method

$\nabla \boldsymbol{F} \quad=\quad$ Jacobian of the error vector with respect to free variables

$f \quad=\quad$ vector field of the elliptic Hill problem with ellipsoidal Phobos

G $\quad$ = $9+$

g $\quad=$ Phobos gravity vector in the pulsating synodic reference frame, $\left[g_{x}, g_{y}, g_{z}\right]^{T}$

$H \quad=$ spacecraft altitude across quasi-satellite orbits $(\mathrm{km})$

$=\mathrm{LQR}$ cost function

$J$
$K$

$=$ time-varying solutic the differential Riccati equation

$\begin{array}{lll}k & = & \text { integer index of Fourier series } \\ N & = & \text { number of quasi-periodic trajectories for numeri- }\end{array}$ cal continuation procedure
$N_{1}$

$P, R$

$R$

$\boldsymbol{R}$

$r$

$s$

$T$

$\boldsymbol{u}$

$\boldsymbol{v}=\left[\begin{array}{lll}\dot{x} & \dot{y} & \dot{z}\end{array}\right]^{T}$

X

X

$\hat{\boldsymbol{X}}$

$\boldsymbol{X}^{*}$

Z

$\bar{\alpha}, \bar{\beta}, \bar{\gamma}$

$\delta s$

$\epsilon$

$\Theta$

Received 20 December 2019; revision received 24 October 2020; accepted for publication 30 October 2020; published online XX epubMonth XXXX. Copyright $\odot 2020$ by the authors. Published by the American Institute of Aeronautics and Astronautics, Inc., with permission. All requests for copying and permission to reprint should be submitted to CCC at www.copyright.com; employ the eISSN 1533-3884 to initiate your request. See also AIAA Rights and Permissions www.aiaa.org/randp.

*Surrey Research Fellow, Surrey Space Centre, Guildford.

SPS Postdoctoral Fellow, Institute of Space and Astronautical Science, anagawa.

MMX Project Engineer, Institute of Space and Astronautical Science, agawa.

${ }^{\S}$ Full Professor, Institute of Space and Astronautical Science, Kanagawa.
$=$ number of $\theta$ intervals for two-dimensional torus maps

$=$ relative state and control weight matrices

$=$ rotation operator

$=$ spacecraft dimensional position vector with respect to Phobos principal axis frame, $[X, Y, Z]^{T}$ $(\mathrm{km})$

$=$ spacecraft position vector in pulsating synodic reference frame, $[x, y, z]^{T}$

$=$ family branch arclength

$=$ stroboscopic time

$=$ linear quadratic regulator LQR control polic vector in pulsating synodic coordinates

$=$ spacecraft velocity vector in pulsating synodic reference frame [-]

$=$ spacecraft state vector in pulsating normalized synodic coordinates, $\left[\boldsymbol{r}^{T}, \boldsymbol{v}^{T}\right]^{T}$

$=$ spacecraft state vector in dimensional coordinates, $\left[\begin{array}{rr}\tilde{\boldsymbol{r}}^{T}, & \tilde{\boldsymbol{v}}^{T}\end{array}\right]^{T}(\mathrm{~km}, \mathrm{~km} / \mathrm{s})$

$=$ estimated spacecraft state vector in normalized pulsating coordinates

$=$ reference state vector in normalized pulsating coordinates generated via two-dimensional torus maps

$=$ vector of Newton's method free variables

$=$ Phobos largest, intermediate, and smallest ellipsoidal axes $(\mathrm{km})$

$=$ continuation step-length

$=$ cubic root of the primaries mass ratio

$=$ mapping matrix

$=$ torus first angular coordinate (rad)

$=$ estimated torus first angular coordinate at orbit determination epoch ( $\mathrm{rad})$

$=$ normalizing factor $\left(\mathrm{s}^{2} / \mathrm{km}\right)$

$=$ spacecraft longitude with respect to the antiMars direction (rad)

$=$ estimated spacecraft longitude (rad)

$=$ maximum eigenvalue of $C$

$\begin{array}{ll}\nu, M & =\text { Phobos true and mean anomalies (rad) } \\ \hat{\nu} & =\text { Phobos true anomaly at orbit determination }\end{array}$ epoch (rad) 
$\rho$

$\Upsilon$

$\Phi$

$\boldsymbol{\varphi}_{T}$

$\mathcal{X}_{i, k}$

$\chi_{i}$

$\boldsymbol{\Omega}$

*
$=$ rotation number of quasi-periodic invariant tori (rad)

$=$ Phobos kinematic libration angle (rad)

$=$ state transition matrix

$=$ vector image of stroboscopic mapping

$=$ eigenvalues of Floquet matrix

$=$ eigenvalues of a quasi-periodic torus

$=$ Phobos orbital angular velocity vector $(\mathrm{rad} / \mathrm{s})$

$=$ Phebos'sellipsoidal gravity vector $\left(\mathrm{km} / \mathrm{s}^{2}\right)$

\section{Introduction}

D ESPITE several scientific speculations regarding the formation and origin of the Martian moons, Phobos and Deimos, planetary satellites around Mars remain mysterious objects of the solar system [1-3]. On the one hand, the near-equatorial near-circular orbits of these remote bodies suggest that Phobos and Deimos are likely the product of a giant impact between a protoplanetary object and Mars' ancestor [4]. On the other hand, the spectral and geophysical features observed by several spacecraft missions indicate that both Martian moons are extremely dark asteroids captured by the gravity well of the red planet [5].

The Soviet's Phobos was the first exploration program aimed at characterizing the surface of the Martian satellite in the late 1980s [6]. Efforts to visit the Martian moons were briefly resumed in 2011, when the Russian Phobos-Grunt mission was launched to land on Phobos but ended in failure [7]. Although Roscomos is planning to repeat this mission as Phobos-Grunt-2 [7], ESA has recently proposed PHOOTPRINT, an effort to collect material from the surface of Phobos by the end of the next decade [8]. The same agency had also considered, but eventually did not select, DePhine [9], an orbiter to explore both Phobos and Deimos within a similar time frame. As for NASA, three missions to the moons of Mars were proposed during the 2014 Discovery program, although none were eventually selected: PADME [10], which was to perform a number of flybys around each moon; MERLIN [11], which was to flyby Deimos and then orbit and land on Phobos; and PANDORA [12], another moons' orbiter. In recent history, a number of Martian probes have observed and photographed its moons, including NASA's Mariner and Viking orbiters, the Mars Global Surveyor (MGS), and the Mars Reconnaissance Orbiter (MRO) [6]. The ongoing Mars Odyssey of NASA, ISRO's Mars Orbiter Mission (MOM) [13], and ESA's Mars Express (MEX) [14] have also contributed to our most recent understanding and knowledge of the Martian moons. In particular, MEX has provided the most accurate observations of Phobos to date, leading to the latest estimates of its shape, gravity, and ephemeris models.

The latest proposal to explore the Martian satellites is the Martian Moons eXploration (MMX) mission, an international effort lead by the Japanese Aerospace Exploration Agency (JAXA). The goal of the MMX mission is to settle the debate on the moons' origin by retrieving pristine samples from the surface of Phobos [15]. The samples are planned to be collected in 2027 after extensive observation campaigns carried out from low-altitude retrograde orbits around the Martian satellite [16].

The study of retrograde relative trajectories around the secondary of two attracting masses has been of interest to astronomers and orbital mechanicists since their original discovery in 1897 [17]. Already in 1913, Jackson was theorizing that this type of orbits may be more stable than direct trajectories [18], a result that was eventually confirmed by Hénon in his numerical investigations of the restricted three-body problem [19]. Based on the results of Hénon's analysis, retrograde relative orbits, also known in the literature as distant retrograde orbits or quasi-satellite orbits, have gained the attention of several space agencies and astrodynamics researchers looking for efficient trajectories to explore the solar system (e.g., [20-22]). Of particular relevancy to this work are the contributions by Lidov and Vashkov'yak [23], Gil and Schwartz [24], and Zamaro and Biggs [25], who first demonstrated how the eccentricity and irregular gravity field of Phobos can play a significant role in governing the dynamic evolution of satellites near the surface of the Martian moon. In spite of these efforts, work in the literature remains primarily focused on isolated periodic orbits whose period is resonant with the orbital period of Phobos around Mars [26]. Only recently, Scheeres et al. [27] have discussed about the possibility of replacing purely periodic quasi-satellite orbits (QSOs) with families of quasi-periodic invariant tori (QPT) that offer more accuracy and insight into the elliptic Hill problem of Phobos. In [27], however, quasi-periodic trajectories are calculated without a robust and appropriate continuation procedure on the eccentricity of the Martian satellite. Furthermore, questions remain on how candidate solutions could be properly maintained in light of 1) mission analysis tools enabled by QPT and 2) operational constraints driven by orbit determination and maneuver scheduling. Both of these issues are addressed in this research.

In general, the problem of maintaining a desired spacecraft trajectory is tackled by either exploiting the chaotic dynamics of the threebody problem with dynamic system theory techniques or via optimal control methods $[28,29]$. The Floquet mode approach and Hamiltonian structure-preserving strategies belong to the first class of orbit maintenance problems, where impulsive maneuvers are typically generated based on the unstable mode or component of reference periodic orbits [30-32]. Because of the inherent linear stability of QSOs, however, neither of these two approaches seem suitable for the station-keeping analyses of MMX. Alternatively, one could consider optimal control methods such as the optimal continuation strategy (OCS) being used for the THEMIS-ARTEMIS satellites [33]. OCS solves two-point boundary value problems that constrain the state of the spacecraft at downstream surface of section crossings (e.g., the primaries' orbital plane for three-dimensional halo orbits). This strategy does not track a reference trajectory and could generate locally optimal solutions in the real-ephemeris model of the Martian system. However, it relies on the solution of large and highly nonlinear optimization problems that could become a challenge for the ground-based operations of fast relative trajectories around Phobos. In fact, knowing that MMX would complete several revolutions around Phobos per day, it is preferable to use less sophisticated guidance laws that can quickly generate suboptimal control inputs based on the current knowledge of the spacecraft state. The Target Point Approach (TPA), LQR controllers, and ZEM/ZEV guidance laws all fall under this category. The TPA offers an analytical expression for impulsive maneuvers that minimizes the relative position error between the spacecraft and a reference trajectory at arbitrarily specified downstream points $[34,35]$. Similar to OCS, the TPA has also been tested in space and it is now being considered for upcoming CubeSat missions like LUMIO and EQUULEUS [36,37]. In [38], the performances of an infinite-horizon LQR and a disturbance rejection control are compared for minimizing the station-keeping costs of several halo orbits in the sun-Earth and Earth-moon circular problems. In [39-42], ZEM/ZEV guidance laws are tested under three-body dynamics and eventually refined via machine learning algorithms. Inverse reinforcement learning and apprenticeship learning have also been applied with success to the problem of stationkeeping of near-rectilinear halo orbit in the Earth-moon system [43]. More recently, Kolmogorov-Arnold-Moser theory has been applied to construct reference trajectories that are naturally bounded and thus facilitate orbit maintenance for the case of quasi-periodic solutions in higher-fidelity models [44]. Using the nonlinear output regulation theory, a general controller has been derived to achieve station-keeping of quasi-periodic invariant tori [45]. All of these approaches can be considered for the MMX mission, pending the careful design and selection of high-fidelity reference trajectories that would minimize linearization errors and accurately predict the evolution of MMX around Phobos.

This paper presents the orbit design and maintenance strategy of the MMX mission in the framework of the elliptic Hill problem with ellipsoidal Phobos. The resulting nonautonomous system is populated by families of quasi-periodic invariant tori that are generated through a numerical continuation procedure on stroboscopic mappings. Five baseline trajectories are selected with different altitude profiles to meet the scientific and engineering requirements of MMX. Stability and sensitivity analyses are carried out in order to investigate the dynamic features of high-fidelity QSOs and identify optimal injection points. Each of the candidate trajectories is tested under 
navigation, insertion, and execution errors to verify the operational feasibility of the newly found solutions. Orbits are maintained with a time-varying LQR controller that selects appropriate reference trajectories based on two-dimensional (2D) torus maps and the estimated longitude of the spacecraft at the start of a maneuver. Future work will explore the advantages and disadvantages of the LQR methodology over TPA and ZEM/ZEV approaches based on the proposed reference trajectory selection method. As a result, the main contributions and innovation of this work can be summarized as follows: A predictorcorrector technique is introduced to systematically generate quasiperiodic invariant tori in the elliptic Hill problem of an ellipsoidal Phobos. Orbits designed in this high-fidelity model are later considered for real mission scenarios and could be easily transitioned in the full-ephemeris model of the Martian system [46]; 2D torus maps are engineered to 1) characterize the sensitivity of high-fidelity QSO with respect to realistic orbit determination errors and 2) remove phasing errors in orbit maintenance analyses. A time-varying LQR controller is implemented in order to assess the operational feasibility of candidate MMX baseline trajectories in spite of navigation and injection errors.

The results and major findings of our analyses are illustrated in the following. Section II presents the equations of motion and the model assumptions adopted for this work. Section III recalls the numerical procedure used for the continuation of quasi-periodic trajectories over the eccentricity of the Martian moon. The results of our numerical continuation technique are illustrated in Sec. IV, along with 2D torus maps that portray altitude and spacecraft longitudes across the five baseline trajectories of MMX. The stability of the newly found solutions is also discussed, along with the sensitivity analysis of the nominal QSOs (Sec. V). In Sec. VI, orbits are maintained with a timevarying LQR controller and tested under injection, navigation, and execution errors via Monte Carlo simulations. Conclusions and final remarks are drawn in Sec. VII.

\section{Dynamic Model}

Consider the motion of a mass particle subject to the gravitational attraction of a spherical Mars and a constant-density tri-axial ellipsoid representing Phobos. The equations of motion are better expressed in a rotating reference frame centered on the Martian moon and such that $\hat{\boldsymbol{x}}$ is constantly aligned with the Mars-Phobos direction, pointing away from Mars, $\hat{z}$ is parallel to the orbital angular momentum of Phobos, and $\hat{\boldsymbol{y}}$ completes the frame, $\hat{\boldsymbol{y}}=\hat{\boldsymbol{z}} \times \hat{\boldsymbol{x}}$ (see Fig. 1). The motion of the barycenter of Phobos is assumed to be Keplerian despite the asymmetric and irregular gravity field exerted on Mars. It is also known that the Martian satellite is in a tidal-locked configuration [25], completing one revolution around its maximum axis of inertia in the time span of an orbital period around Mars. Accordingly, the rotational spin rate of Phobos and the angular velocity of the reference frame may be approximated as $\boldsymbol{\omega}_{P}=n_{P} \hat{z}$ and $\boldsymbol{\Omega}=n_{P}\left(1-e_{P}^{2}\right)^{-3 / 2} \gamma^{2} \hat{z}$, respectively, where $\gamma=\left(1+e_{P} \cos \nu\right), \nu$ is the true anomaly of Phobos with respect to Mars, $n_{P}$ is Phobos mean motion, and $e_{P}$ is its eccentricity. Relevant quantities of Phobos are tabulated in Table 1 based on the numerical values provided in [47]. In view of the small Phobos-to-Mars mass ratio and the low altitude envisaged for the

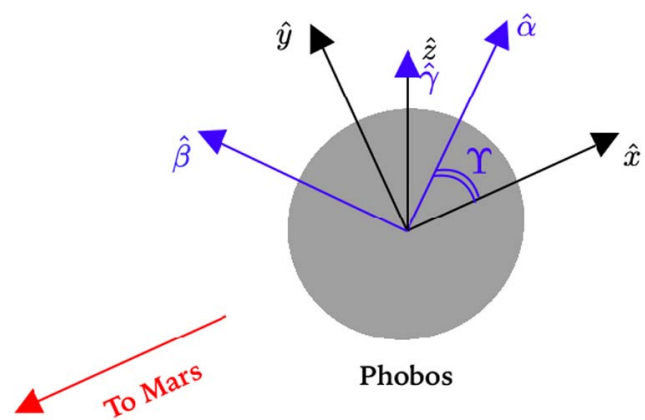

Fig. 1 Hill reference frame and kinematic libration angles for the MarsPhobos system.
Table 1 Phobos's physical parameters [47]

\begin{tabular}{lccc}
\hline \hline Symbol & Quantity & Value & Unit \\
\hline $\bar{\alpha}$ & Largest semimajor axis & 13.03 & $\mathrm{~km}$ \\
$\bar{\beta}$ & Intermediate semimajor axis & 11.40 & $\mathrm{~km}$ \\
$\bar{\gamma}$ & Smallest semimajor axis & 9.14 & $\mathrm{~km}$ \\
$\sigma_{P}$ & Density & 1.860 & $\mathrm{~g} / \mathrm{cm}^{3}$ \\
$M_{P}$ & Mass & $1.058 \times 10^{16}$ & $\mathrm{~kg}$ \\
$\mu_{P}$ & Gravitational parameter & $7.060 \times 10^{-4}$ & $\mathrm{~km}^{3} / \mathrm{s}^{2}$ \\
$a_{P}$ & Phobos's orbit semimajor axis & 9377 & $\mathrm{~km}$ \\
$e_{P}$ & Phobos's orbit eccentricity & 0.0151 & - \\
$P_{P}$ & Rotational period & 7.66 & $\mathrm{~h}$ \\
$n_{P}$ & Mean motion/spin rate & $2.279 \times 10^{-4}$ & $\mathrm{rad} / \mathrm{s}$ \\
\hline \hline
\end{tabular}

proximity operations of MMX, the dynamics of the system can be well approximated via the equations of the elliptic Hill problem [27]:

$$
\ddot{x}-2 \dot{y}=g_{x}+\frac{3 x}{\gamma}, \quad \ddot{y}+2 \dot{x}=g_{y}, \quad \ddot{z}=g_{z}-z
$$

where dots denote differentiation with respect to Phobos's true anomaly, $\boldsymbol{X}=[x, y, z, \dot{x}, \dot{y}, \dot{z}]^{\mathrm{T}}$ is the state of MMX in pulsating normalized coordinates, and $\boldsymbol{g}=\left[g_{x}, g_{y}, g_{z}\right]^{\mathrm{T}}$ is the normalized acceleration due to the attracting ellipsoidal mass. Pulsating normalized coordinates are related to dimensional variables via

$$
\tilde{\boldsymbol{r}}=\epsilon p_{P} \gamma^{-1} \boldsymbol{r}, \quad \tilde{\boldsymbol{v}}=\epsilon p_{P} \gamma^{-1}\left(\boldsymbol{v}+e_{P} \sin \nu \gamma^{-1} \boldsymbol{r}\right)
$$

where $\boldsymbol{r}=[x, y, z]^{\mathrm{T}}, \boldsymbol{v}=[\dot{x}, \dot{y}, \dot{z}]^{\mathrm{T}}, \epsilon=\left(\mu_{P} / \mu_{M}\right)^{1 / 3}$ is the cubic root of the Phobos-Mars mass ratio, $p_{P}=a_{P}\left(1-e_{P}^{2}\right)$ is the semilatus rectum of Phobos's orbit, and $a_{P}$ is its orbit semimajor axis. Accordingly,

$$
\boldsymbol{g}=\kappa R_{z}[\Upsilon] \boldsymbol{G}
$$

where $\kappa=p_{P}^{2} \epsilon^{-1} \mu_{M}^{-1} \gamma^{-3}, R_{z}[\Upsilon]$ is the rotation matrix about the $\hat{z}$ axis and given by

$$
R_{z}[\Upsilon]=\left[\begin{array}{ccc}
\cos \Upsilon & \sin \Upsilon & 0 \\
-\sin \Upsilon & \cos \Upsilon & 0 \\
0 & 0 & 1
\end{array}\right]
$$

and $\Upsilon=\nu-M$ is the kinematic libration angle defined in Fig. 1 and given by the instantaneous difference between Phobos's true and mean anomalies. It is assumed that $\gamma$, the unit vector along the smallest axis of Phobos, remains parallel to $\hat{z}$. As a result, the kinematic libration angle can be also defined as the instantaneous angle between $\boldsymbol{\alpha}$ and $\hat{\boldsymbol{x}}$, or $\boldsymbol{\beta}$ and $\hat{\boldsymbol{y}}$, where $\boldsymbol{\alpha}$ and $\boldsymbol{\beta}$ denote the direction of Phobos's largest and intermediate axes, respectively. The vector $\boldsymbol{G}$ stands for the gravitational acceleration due to an ellipsoidal Phobos and its components are provided in the principal axes frame of the Martian moon by [48]

$$
\begin{aligned}
G_{x} & =-\frac{3}{2} \mu_{P} X \int_{0}^{\infty}\left(\frac{1}{\bar{\alpha}^{2}+\Lambda+l}\right) \frac{\mathrm{d} l}{\Delta(l+\Lambda)} \\
G_{y} & =-\frac{3}{2} \mu_{P} Y \int_{0}^{\infty}\left(\frac{1}{\bar{\beta}^{2}+\Lambda+l}\right) \frac{\mathrm{d} l}{\Delta(l+\Lambda)} \\
G_{z} & =-\frac{3}{2} \mu_{P} Z \int_{0}^{\infty}\left(\frac{1}{\bar{\gamma}^{2}+\Lambda+l}\right) \frac{\mathrm{d} l}{\Delta(l+\Lambda)}
\end{aligned}
$$

where $\boldsymbol{R}=[X, Y, Z]^{\mathrm{T}}=R_{z}[-\Upsilon] \tilde{\boldsymbol{r}}=\epsilon p_{P} \gamma^{-1} R_{z}[-\Upsilon] \boldsymbol{r}$ is the dimensional position vector of the satellite as seen from the principal-axis frame of Phobos. Furthermore,

$$
\Delta(l+\Lambda)=\sqrt{\left(\bar{\alpha}^{2}+\Lambda+l\right)\left(\bar{\beta}^{2}+\Lambda+l\right)\left(\bar{\gamma}^{2}+\Lambda+l\right)}
$$


where $\bar{\alpha}, \bar{\beta}$, and $\bar{\gamma}$ stand for the largest, intermediate, and smallest semimajor axes of the Martian moon; $\mu_{P}$ is the gravitational parameter of the Martian moon; and $\Lambda$ is the positive root of

$$
\frac{X^{2}}{\bar{\alpha}^{2}+\Lambda}+\frac{Y^{2}}{\bar{\beta}^{2}+\Lambda}+\frac{Z^{2}}{\bar{\gamma}^{2}+\Lambda}-1=0
$$

Also notice that the elliptic integrals appearing in Eq. (5) can be rapidly approximated using the numerical procedures of Ref. [49]. Similar techniques can be used to calculate the partial derivatives of Eq. (5) and enable the integration of the state transition matrix along arbitrary trajectories.

\section{Numerical Continuation of 2D Invariant Tori}

The equations of motion in Eq. (1) can be rewritten as a system of first-order ordinary differential equations via

$$
\dot{\boldsymbol{X}}=\boldsymbol{f}\left(\nu, \boldsymbol{X}, e_{P}\right)
$$

We express the dependency of the vector field $f$ on $\nu$ and $e_{P}$ to highlight that the system (8) is nonautonomous and depends on the constant parameter $e_{P}$. Accordingly, periodic orbits are isolated at best and cannot be used to fully organize the system dynamics of the elliptic Hill problem. Conversely, quasi-periodic trajectories that densely cover the surface of 2D invariant tori remain organized in families, similarly to periodic orbits of time-autonomous systems like the circular restricted three-body problem [27]. These higher-dimensional manifolds are better equipped to describe the dynamics of the system in the vicinity of Phobos, as well as to provide new and accurate initial guesses for the trajectory design of the MMX mission [46].

To calculate quasi-periodic trajectories, we modify the GMOS algorithm outlined in Refs. $[50,51]$ to slowly grow the eccentricity of Phobos from 0 to $e_{P}=0.0151$. Starting from $e=0$, we pick an altitude and a periodic orbit that exists in the circular problem, close to the desired trajectory of MMX. Let $\boldsymbol{X}_{0}$ be the initial state of such a periodic orbit with $T$ as its orbital period. Next, we pick $N$ equally spaced values of Phobos's true anomaly, i.e., $\nu_{i}=2 \pi(i-1) / N$, $i=1, \ldots, N$, and slightly increase the value of the eccentricity by a step of $e=10^{-4}$ to introduce time-varying effects. A curve of candidate initial conditions can be now constructed from $\boldsymbol{X}_{0, i}=$ $\left[\left(1-e^{2}\right) /\left(1+e \cos \nu_{i}\right)\right] \boldsymbol{X}_{0}$ so as to account for deviations with respect to the circular case. The collection of these points, namely, $\left\{\boldsymbol{X}_{0, i}\right\}$, is a discrete representation of a curve $\mathcal{C}(\nu) \in \mathbb{R}^{6}$ that shall be invariant for the system dynamics (8). In particular, we expect the images of the stroboscopic mapping $\left\{\boldsymbol{\varphi}_{T}\left(\boldsymbol{X}_{0, i} ; \nu_{i}, e\right)\right\}$ to satisfy the quasi-periodic boundary conditions given by [50]

$$
R_{-\rho}\left[\left\{\boldsymbol{\varphi}_{T}\left(\boldsymbol{X}_{0, i} ; \nu_{i}, e\right)\right\}\right]-\left\{\boldsymbol{X}_{0, i}\right\}=\mathbf{0}_{N}
$$

where $R_{-\rho}[\cdot]$ is a rotational operator that undoes the changes in true anomaly after one orbital period $T$, and $\rho$ is the rotation number of the quasi-periodic invariant torus, i.e., the angular displacement between any initial condition $\boldsymbol{X}_{0, i}$ and its stroboscopic image after one stroboscopic time $T$, i.e., $\boldsymbol{\varphi}_{T}\left(\boldsymbol{X}_{0, i} ; \nu_{i}, e\right)$ on $\mathcal{C}(\nu) \in \mathbb{R}^{6}$. Because dots denote differentiation with respect to Phobos's true anomaly, it can be demonstrated that $\rho=i T=T$. As a result, Eq. (9) is a system of $6 \times N$ equations in the $6 \times N+2$ unknowns $\boldsymbol{Z}=\left(\left\{\boldsymbol{X}_{0, i}\right\}, T, e\right)$. To find quasi-periodic solutions for increasing values of $e$, we augment the system (9) with phase conditions and parameterizing equations that allow us to uniquely update the initial values of $\boldsymbol{Z}$ via Newton's method:

$$
\boldsymbol{Z}^{k+1}=\boldsymbol{Z}^{k}-\nabla \boldsymbol{F}\left(\boldsymbol{Z}^{k}\right)^{\dagger} \boldsymbol{F}\left(\boldsymbol{Z}^{k}\right)
$$

where $\boldsymbol{F}\left(\boldsymbol{Z}^{k}\right)$ is the error vector given by

$$
\boldsymbol{F}(\boldsymbol{Z})=\left[\begin{array}{c}
R_{-T}\left[\left\{\boldsymbol{\varphi}_{T}\left(\boldsymbol{X}_{0, i} ; \nu_{i}, e\right)\right\}\right]-\left\{\boldsymbol{X}_{0, i}\right\} \\
p . c\left(\left\{\boldsymbol{X}_{0, i}\right\}\right) \\
s_{1}(T) \\
s_{2}\left(\left\{\boldsymbol{X}_{0, i}\right\}, T, e\right)
\end{array}\right]
$$

and $\nabla \boldsymbol{F}\left(\boldsymbol{Z}^{k}\right)^{\dagger}$ is the left pseudo-inverse of its Jacobian matrix with respect to the free variables $\boldsymbol{Z}$. Explicit expressions for $\nabla \boldsymbol{F}\left(\boldsymbol{Z}^{k}\right)$ can be found in Ref. [51], except for the partial derivatives with respect to $e$ that are hereby reported.

The phase conditions and parameterizing equations in Eq. (11) may be given as in [50]:

$$
\begin{gathered}
p . c\left(\left\{\boldsymbol{X}_{0, i}\right\}\right):=\left\langle\left\{\boldsymbol{X}_{0, i}\right\}-\left\{\tilde{\boldsymbol{X}}_{0, i}\right\}, \frac{\partial\left\{\tilde{\boldsymbol{X}}_{0, i}\right\}}{\partial \theta}\right\rangle \\
s_{1}(T):=T-\tilde{T} \\
s_{2}\left(\left\{\boldsymbol{X}_{0, i}\right\}, T, e\right):=\left\langle\boldsymbol{Z}-\tilde{\boldsymbol{Z}}, \frac{\partial \tilde{\boldsymbol{Z}}}{\partial s}\right\rangle-\delta s
\end{gathered}
$$

where

$$
\frac{\partial\left\{\tilde{\boldsymbol{X}}_{0, i}\right\}}{\partial \theta}=\frac{T}{2 \pi}\left(\left\{\boldsymbol{f}\left(\nu, \tilde{\boldsymbol{X}}_{0, i}, e\right)\right\}-\frac{\partial\left\{\tilde{\boldsymbol{X}}_{0, i}\right\}}{\partial \nu}\right)
$$

$\delta s$ is the continuation step length for the pseudo-arclength continuation constraint $s_{2}\left(\left\{\boldsymbol{X}_{0, i}\right\}, T, e\right)$ [52], and tildes denote values calculated from a previously known quasi-periodic solution. For the first quasi-periodic invariant torus, $\left\{\tilde{\boldsymbol{X}}_{0, i}\right\}=\boldsymbol{X}_{0}, \forall i=1, \ldots, N, \tilde{T}=T$, the period of the QSO orbit in the circular problem, and $\tilde{e}=0$. Note that the values of the partial derivatives $\partial\left\{\tilde{X}_{0, i}\right\} / \partial \nu$ can be quickly estimated from the Fourier coefficients of $\mathcal{C}(\nu)$, namely,

$$
\boldsymbol{c}_{k}=\frac{1}{\sqrt{N}} \sum_{i=1}^{N} \boldsymbol{X}_{0, i} \exp \left[\mathrm{i} k \nu_{i}\right]
$$

such that

$$
\boldsymbol{X}_{0}(\nu) \simeq \frac{1}{\sqrt{N}} \sum_{k=-(N-1) / 2}^{(N-1) / 2} \boldsymbol{c}_{k} \exp [-\mathrm{i} k \nu]
$$

and

$$
\frac{\partial \boldsymbol{X}_{0}}{\partial \nu}(\nu) \simeq \frac{1}{\sqrt{N}} \sum_{k=-(N-1) / 2}^{(N-1) / 2}-\mathrm{i} k \boldsymbol{c}_{k} \exp [-i k \nu]
$$

Similarly, if $\boldsymbol{d}_{k}$ are the Fourier coefficients derived from $\left\{\boldsymbol{\varphi}_{T}\left(\left\{\boldsymbol{X}_{0, i}\right\} ; \nu_{i}, e\right)\right\}$, then

$$
R_{-T}\left[\left\{\boldsymbol{\varphi}_{T}\left(\boldsymbol{X}_{0} ; \nu, e\right)\right\}\right] \simeq \frac{1}{\sqrt{N}} \sum_{k=-(N-1) / 2}^{(N-1) / 2} \boldsymbol{d}_{k} \exp [-\mathrm{i} k(\nu-T)]
$$

It follows that the partial derivatives of $R_{-T}\left[\left\{\boldsymbol{\varphi}_{T}\left(\boldsymbol{X}_{0, i} ; \nu_{i}, e\right)\right\}\right]$ with respect to $e$ are given by

$$
\begin{aligned}
\frac{\partial R_{-T}\left[\left\{\boldsymbol{\varphi}_{T}\left(\boldsymbol{X}_{0, i} ; \nu_{i}, e\right)\right\}\right]}{\partial e} & \simeq \frac{\partial}{\partial e}\left\{\frac{1}{\sqrt{N}} \sum_{k=-(N-1) / 2}^{(N-1) / 2}\left[\frac{1}{\sqrt{N}} \sum_{i=1}^{N} \boldsymbol{\varphi}_{T}\left(\boldsymbol{X}_{0, i} ; \nu_{i}, e\right) \exp \left[\mathrm{i} k \nu_{i}\right]\right] \exp [-\mathrm{i} k(\nu-T)]\right\} \\
& \simeq \frac{1}{\sqrt{N}} \sum_{k=-(N-1) / 2}^{(N-1) / 2}\left[\frac{1}{\sqrt{N}} \sum_{i=1}^{N} \frac{\partial \boldsymbol{\varphi}_{T}\left(\boldsymbol{X}_{0, i} ; \nu_{i}, e\right)}{\partial e} \exp \left[\mathrm{i} k \nu_{i}\right]\right] \exp [-\mathrm{i} k(\nu-T)], \\
& \simeq R_{-T}[\{\Theta(T, 0)\}]
\end{aligned}
$$


where $\Theta(T, 0)$ is the mapping matrix obtained from the numerical integration of

$$
\dot{\Theta}=\left[\frac{\partial f}{\partial \boldsymbol{X}}\right] \Theta+\left[\frac{\partial \boldsymbol{f}}{\partial e}\right], \quad \text { subject to } \quad \Theta(0,0)=\mathbf{0}_{6 \times 1}
$$

over one stroboscopic time $T$. The analytical expressions of $[\partial f / \partial X]$ and $[\partial f / \partial e]$ are derived in the Appendix. Also notice that Eqs. (12a) and $(12 b)$ do not depend on the eccentricity of Phobos, whereas $\left(\partial s_{2} / \overline{\partial e}\right)=(\partial \tilde{e} / \partial s)$. This and the other components of the family tangent

$$
\frac{\partial \tilde{\boldsymbol{Z}}}{\partial s}=\left[\left(\frac{\partial \tilde{\boldsymbol{X}}_{0}}{\partial s}\right)^{\mathrm{T}}, \frac{\partial \tilde{T}}{\partial s}, \frac{\partial \tilde{e}}{\partial s}\right]^{\mathrm{T}}
$$

appearing in Eq. (12c) may be obtained as follows. If $\boldsymbol{Z}$ is a solution of Eq. (11), it must nullify the reduced error vector

$$
\mathcal{F}(\boldsymbol{Z})=\left[\begin{array}{c}
R_{-T}\left[\left\{\boldsymbol{\varphi}_{T}\left(\boldsymbol{X}_{0, i} ; \nu_{i}, e\right)\right\}\right]-\left\{\boldsymbol{X}_{0, i}\right\} \\
p . c\left(\left\{\boldsymbol{X}_{0, i}\right\}\right) \\
s_{1}(T)
\end{array}\right]=\mathbf{0}_{6 N+2}
$$

for any value of $e$. Indeed, Eq. (20) defines a one-parameter family of quasi-periodic trajectories with the same stroboscopic time $T$. Let $s$ be the arclength of this family branch and consider small deviations from the current family member $\boldsymbol{Z}(s)$. Up to the first order in $\delta s$,

$$
\mathcal{F}(\boldsymbol{Z}(s+\delta s)) \simeq \mathcal{F}(\boldsymbol{Z}(s))+\nabla \mathcal{F}(\boldsymbol{Z}(s)) \frac{\partial \boldsymbol{Z}}{\partial s} \simeq \mathbf{0}_{6 N+2}
$$

thereby demonstrating that

$$
\frac{\partial \boldsymbol{Z}}{\partial s} \in \operatorname{Ker}[\nabla \mathcal{F}(\boldsymbol{Z}(s))]
$$

Similarly, $\boldsymbol{Z}(s+\delta s) \simeq \boldsymbol{Z}(s)+(\partial \boldsymbol{Z} / \partial s) \delta s$ implies

$$
\left\langle\boldsymbol{Z}(s+\delta s)-\boldsymbol{Z}(s), \frac{\partial \boldsymbol{Z}}{\partial s}\right\rangle \simeq\left\langle\frac{\partial \boldsymbol{Z}}{\partial s}, \frac{\partial \boldsymbol{Z}}{\partial s}\right\rangle \delta s
$$

which reduces to Eq. $(\underline{12 c})$ if and only if

$$
\left\langle\frac{\partial Z}{\partial s}, \frac{\partial Z}{\partial s}\right\rangle=1
$$

Equations (9), (12), (13), (22), and (24) lay out the foundation of the time-nonautonomous GMOS predictor-corrector scheme used in this research for generating families of quasi-periodic trajectories as a function of $e$. Starting from $\left\{\tilde{\boldsymbol{X}}_{0, i}\right\}=\left(1-e^{2} / 1+e \cos \nu_{i}\right) \boldsymbol{X}_{0}$, $\tilde{T}=T, \tilde{e}=10^{-4}, \partial\left\{\boldsymbol{X}_{0, i}\right\} / \partial s=\mathbf{0}_{6 N},(\partial \tilde{T} / \partial s)=0,(\partial \tilde{e} / \partial s)=1$, and $\delta s=10^{-4}$, we march along the family branch until $e=e_{P}=$ 0.0151 , i.e., the eccentricity of Phobos's orbit. Upon convergence, the images of the stroboscopic mappings of the solution points $\left\{\boldsymbol{X}_{0, i}\right\}$ are collected over $N_{1}$ equally spaced values of $t_{i} \in[0, T]$ and rotated by $\rho_{i}=t_{i}$ to generate $2 \mathrm{D}$ torus maps as in Ref. [46]. After numerical experiments, we found that setting $N=75$ and $\bar{N}_{1}=500$ gives the best compromise between the accuracy of quasi-periodic trajectories/ $2 \mathrm{D}$ torus maps and the runtime of the numerical procedure. We also recall that the stability of quasi-periodic invariant tori can be assessed by plotting the eigenvalues of the Floquet matrix (see Refs. [51-55] and references therein for details). The results of these investigations are illustrated in Sec. IV.

\section{Candidate Quasi-Satellite Orbits}

According to the latest baseline, MMX will be launched in September 2024 and inserted into orbit around Mars after 11 months of interplanetary cruise [15]. Upon arrival, the spacecraft will approach the vicinity of Phobos with a sequence of phasing maneuvers and insert into retrograde relative trajectories or QSOs to start its measurement campaign. Five candidate trajectories are currently considered for the proximity phase of MMX. These candidate orbits are labeled as QSO-H, QSO-M, QSO-La, QSO-Lb, and QSO-Lc and their geometrical features are listed in Table 2 [46]. The nomenclature follows the standard adopted by the MMX flight dynamics team and is qualitatively related to the QSO altitude with respect to the surface of Phobos.

As we include the eccentricity of Phobos, each of these candidate trajectories becomes a 2D quasi-periodic torus that is invariant under the system dynamics of Eq. (8). To generate these invariant manifolds, we run the GMOS predictor-corrector scheme of Sec. III, while starting from the positive $x$ axis of the Mars-Phobos rotating frame. The output of the numerical continuation is summarized in Fig. 2 for the high- and medium-altitude QSO, and in Fig. 3 for the low-altitude QSOs. Three plots are shown for each candidate orbit: a polar view of the overall trajectory in rotating coordinates where the orbit envelope is quasi-periodic, the longitude of the satellite, and its altitude profile across the entire domain of the QPT. The longitude and altitude profiles are obtained via

$$
\lambda= \begin{cases}\arctan (-y / x) & y \leq 0, x \geq 0 \\ \pi+\arctan (-y / x) & x<0 \\ 2 \pi+\arctan (-y / x) & y>0, x \geq 0\end{cases}
$$

and $H=\sqrt{\left(x-x_{s s}\right)^{2}+\left(y-y_{s s}\right)^{2}+\left(z-z_{s s}\right)^{2}}$, respectively, where $x_{s s}=\bar{\alpha} \cos \lambda \cos \phi, y_{s s}=\bar{\beta} \sin \lambda \cos \phi$, and $z_{s s}=\bar{\gamma} \sin \phi$ are the coordinates of the subsatellite point, and $\phi$ is the latitude of the spacecraft (almost zero for nearly equatorial quasi-satellite orbits). The values of $\lambda$ and $H$ are plotted on an $N_{1} \times N$ grid of $\theta_{i}=2 \pi t_{i} / T$, $t_{i}=0, t_{1}, \ldots, t_{N_{1}}=T$, and $\nu_{i}=2 \pi i / N, i=0,1, \ldots, N$, torus coordinates, hereby referred to as $2 \mathrm{D}$ torus maps.

By definition, the orbit envelope (blue regions) of Figs. $2 \mathrm{a}$ and $2 \mathrm{~d}$ and Figs. 3a, $3 \mathrm{~d}$, and $3 \mathrm{~g}$, hereafter referred to as the generic invariant set $\mathcal{T} \in \mathbb{R}^{6}$, are invariant for the system dynamics of Eq. (8) if there exist a diffeomorphism $\boldsymbol{u}(\theta, \nu):[0,2 \pi] \times[0,2 \pi] \rightarrow \mathcal{T}$ such that the restriction of the vector field to $\mathcal{T}$, namely, $\left.\boldsymbol{f}\right|_{\mathcal{T}}$, is mapped by the inverse of the differential map of $\boldsymbol{u}$ into the constant vector field on the torus given by

$$
\dot{\theta}=2 \pi / T, \quad \dot{\nu}=1
$$

As a result, quasi-periodic trajectories on the surface of $\mathcal{T}$ can be trivially integrated in torus coordinates and mapped back to phase-space coordinates via $\boldsymbol{u}(\theta, \nu)$. This is a clear advantage of the present QPT approach over other methods that would recalculate quasi-periodic trajectories for different configurations of the MMX-Mars-Phobos system. Instead, our tool enables the mission designer to instantly retrieve the dynamics on the surface of the considered torus from any arbitrary initial conditions $(\hat{\theta}, \hat{\nu})$ in $[0,2 \pi] \times[0,2 \pi]$;. Examples of quasi-periodic trajectories are displayed as black lines in Figs. $2 \mathrm{~b}, 2 \mathrm{c}$, 2e, and $2 \mathrm{f}$ and Figs. 3b, 3c, 3e, 3f, 3h, and 3i, thereby illustrating how nominal quasi-periodic trajectories can be quickly generated for arbitrary values of $\lambda$ and/or $H$. Based on this feature, we have implemented an LQR controller that counters the effects of injection and navigation errors by selecting appropriate reference trajectories at the start of a maneuver. The LQR approach is further discussed in Sec. VI after a sensitivity analysis of the computed quasi-periodic solutions.

Table 2 MMX candidate science orbits

\begin{tabular}{cccc}
\hline \hline Name & $X \times Y(\mathrm{~km})$ & $\dot{X} \times \dot{Y}(\mathrm{~m} / \mathrm{s})$ & $T(\mathrm{~h})$ \\
\hline QSO-H & $100 \times 198.47$ & $45.74 \times 22.95$ & 7.59 \\
QSO-M & $50 \times 94.41$ & $23.41 \times 12.04$ & 7.13 \\
QSO-La & $30 \times 48.83$ & $15.31 \times 8.68$ & 5.76 \\
QSO-Lb & $22 \times 30.81$ & $12.79 \times 8.25$ & 4.40 \\
QSO-Lc & $20 \times 26.69$ & $12.31 \times 8.31$ & 3.97 \\
\hline \hline
\end{tabular}




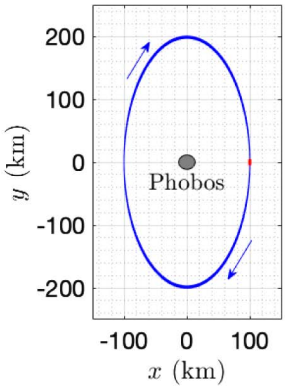

a) QSO-H

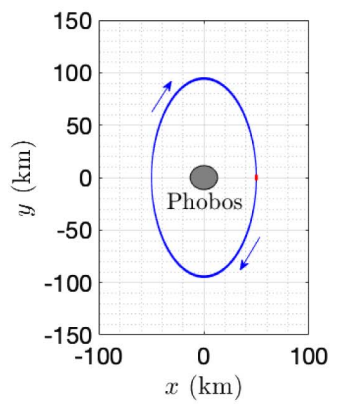

d) QSO-M

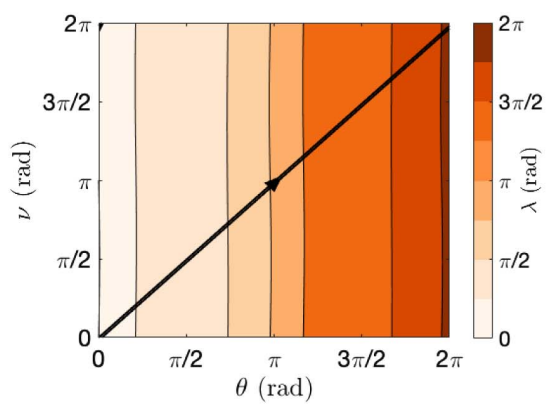

b) QSO-H, longitude

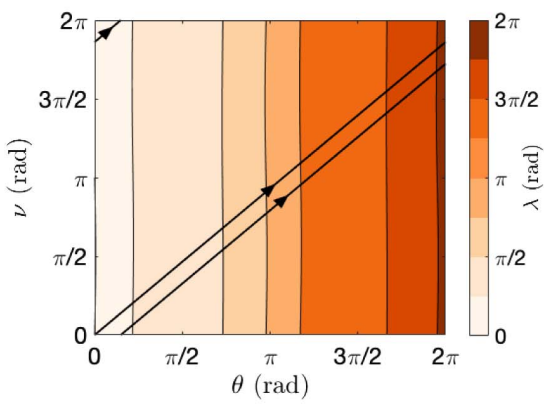

e) QSO-M, longitude

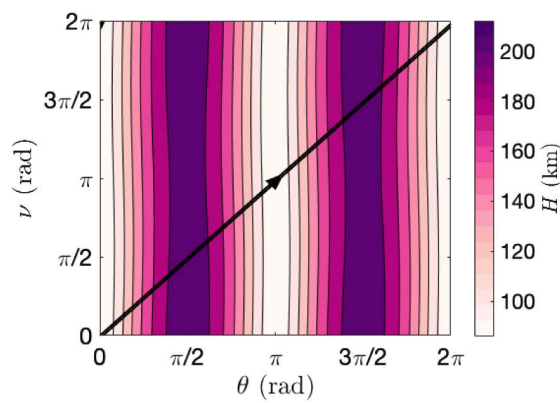

c) QSO-H, altitude

Fig. 2 Mid- and high-altitude QSOs obtained with the GMOS predictor-corrector scheme in the elliptic Hill problem.

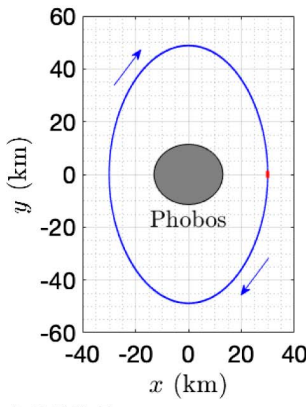

a) QSO-La

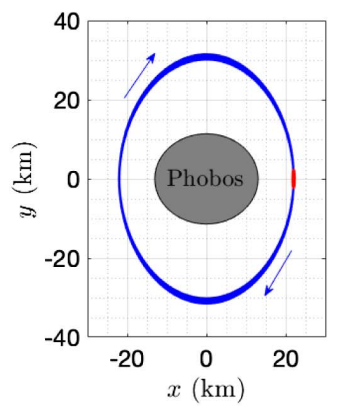

d) QSO-Lb

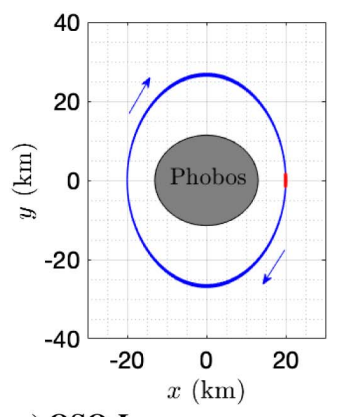

g) QSO-Lc

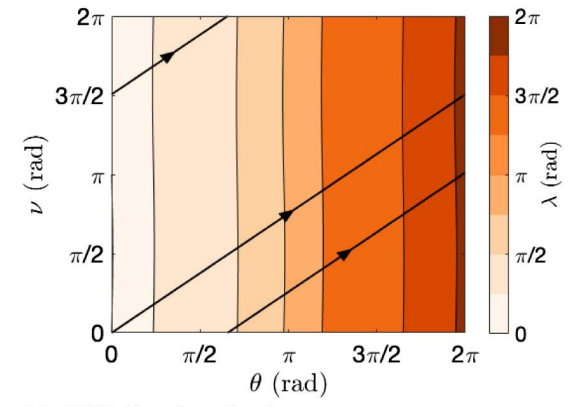

b) QSO-La, longitude

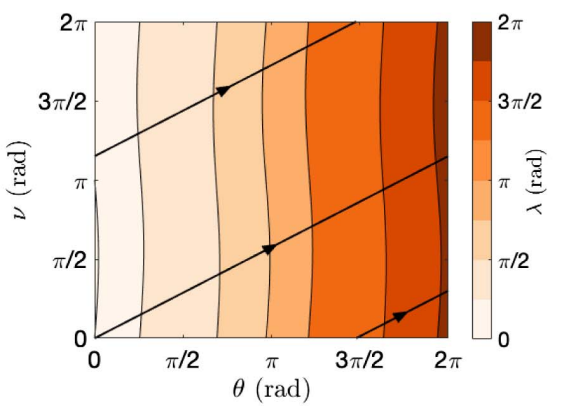

e) QSO-Lb, longitude

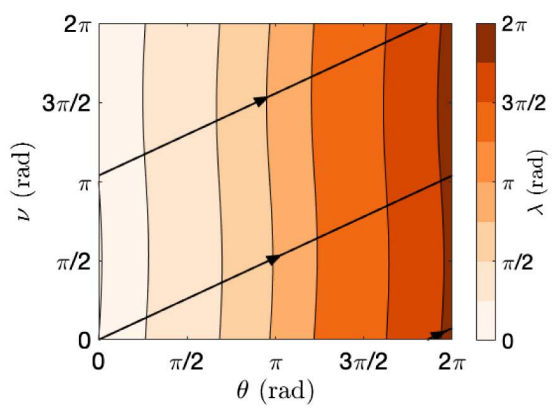

h) QSO-Lc, longitude

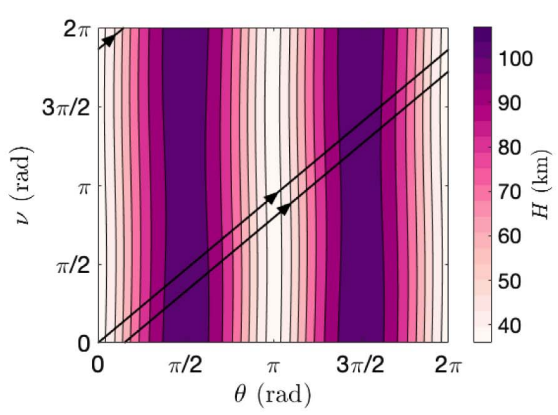

f) QSO-M, altitude 
Finally, Fig. 4 illustrates the eigenvalues of the Floquet matrices extracted from the final iteration of the differential corrector of Sec. III. The interested reader may find additional information on how to gauge the stability properties of a quasi-periodic invariant torus in Refs. [51,53-55]. For the purpose of this paper, note that the $6 N$ eigenvalues of a Floquet matrix, namely, $\mathcal{X}_{i, k}$, are related to the six eigenvalues of a quasi-periodic invariant torus, $\chi_{i}$, via

$$
\mathcal{X}_{i, k}=\chi_{i} e^{\mathrm{i} k \rho}, \quad k=-\frac{N-1}{2}, \ldots, 0, \ldots, \frac{N-1}{2}
$$

Accordingly and pending numerical instabilities in the eigenvalue calculation, all of the $\mathcal{X}_{i, k}$ must be distributed along concentric circles of the complex plane. In the case of the retrograde relative trajectories considered in this research, all of the eigenvalues lie on concentric circles with unitary magnitude. It follows that the considered quasisatellite orbits retain linear stability also when the eccentricity of Phobos is taken into account.

\section{Sensitivity Analysis}

In this section, we analyze the sensitivity of the computed quasisatellite orbits to insertion errors. This investigation provides valuable insight on the selection of possible insertion locations along the baseline QSOs. We consider a uniform grid of $100 \times 100$ torus coordinates in $[0,2 \pi] \times[0,2 \pi]$; and propagate the corresponding initial conditions for one day, i.e., the envisaged operation cycle of MMX. For each of these quasi-periodic trajectories, we calculate the corresponding state transition matrix $\Phi$ and report the maximum eigenvalue $\mu$ of the Cauchy-Green tensor given by $C=\Phi^{\mathrm{T}} \Phi$. Note that the maximum eigenvalue of the Cauchy-Green tensor is typically associated with the finite-time Lyapunov exponent of a trajectory, and it is a good indicator of stability in nonautonomous dynamic systems [56]. In practice, a larger $\mu$ indicates a region where dispersion errors in the phase space grow quicker, and thus a region where MMX should be less preferably injected.

Figure 5 illustrates the values and isolines of the maximum eigenvalue of the Cauchy-Green tensor as a function of the torus coordinates $\theta$ and $\nu$ for the five-candidate QSOs. The values of $\mu$ generally increase for lower-altitude QSOs, indicating that smaller and faster quasi-satellite orbits are more susceptible to injection and navigation errors than higher-altitude QSOs. Also, it appears as insertion along the positive or negative $y$ axis, i.e., $\lambda= \pm \pi / 2$, would minimize the growth of the relative error with respect to the desired spacecraft trajectories. Such a dispersion does not seem to vary greatly with the true anomaly of Phobos except for QSO-H (see Fig. 5a). A possible explanation for this phenomenon could be that the orbital period of higher-altitude QSOs is very close to be in a 1:1 resonance with the orbital period of Phobos. Nevertheless, we can imagine a nominal scenario in which MMX will aim at inserting into quasi-satellite orbits above the leading or trailing edge of Phobos. After insertion, MMX should remain on the targeted quasi-periodic invariant torus for about a month and perform scientific observations, even though the effects of navigation and model uncertainties will gradually drive the spacecraft away from its nominal trajectory. In some cases, we noted that the spacecraft would either escape or impact against the surface of the Martian moon despite the verified linear stability of the candidate QSO. In the next section, we will introduce an LQR-based strategy that mitigates the effects of knowledge errors and thus enables the proximity operations of MMX.

\section{Orbit Maintenance of Quasi-Satellite Orbits}

In this section, we present an LQR strategy for the proximity operations of MMX that leverages the dynamic properties of quasiperiodic invariant tori to overcome the limitation of standard orbit maintenance approaches. In a first stage, efforts have been directed toward the development and implementation of station-keeping strategies using the TPA. Among other strategies, the TPA has shown promising results for the orbit maintenance of periodic orbits in the Earth-moon and sun-Earth systems. The TPA aims at minimizing the impulsive cost of a maneuver based on the current knowledge of the spacecraft state, as well as of the linearly predicted relative error with respect to a nominal reference trajectory at user-defined downstream points. This approach works well as long as the predicted relative errors are within the linearization regime of the reference trajectory. The validity of the linear approximation, which is pivotal for the TPA, ceases to hold for the Mars-Phobos cases, where QSOs complete several revolutions in 1 day (ranging from 3.15 to 6 revolutions in a day for the QSO-H and QSO-Lc cases). During this time frame the actual and nominal trajectories of the spacecraft deviate significantly. Simulations have shown that the largest deviations occur in the longitude with respect to Phobos, as expected from the observed linear stability of the computed manifolds. This has two main consequences: 1) the actual relative error grows beyond the linear regime and cannot be compensated based on the state transition

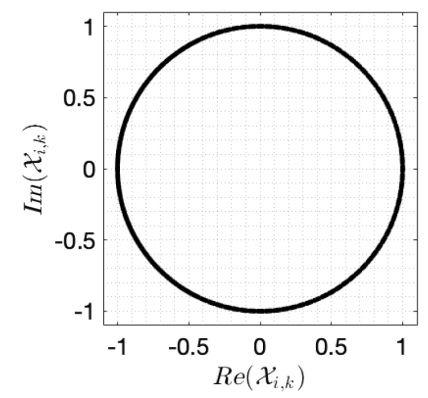

a) QSO-H

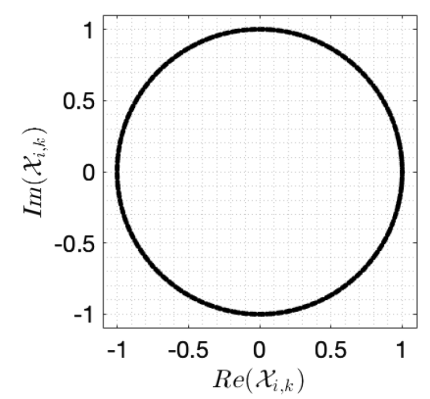

b) QSO-M

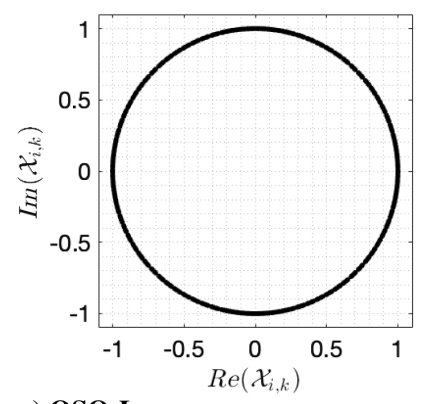

c) QSO-La

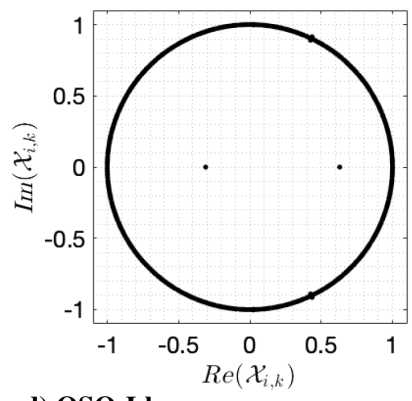

d) QSO-Lb

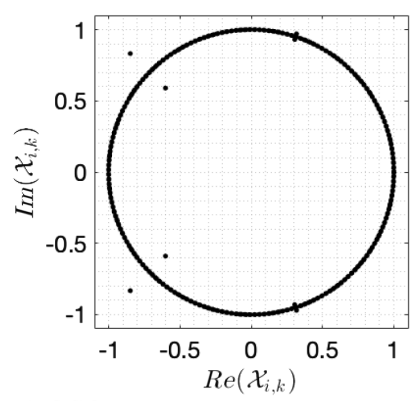

e) QSO-Lc

Fig. 4 Eigenvalues of the Floquet matrices associated with each of the candidate QSOs. 


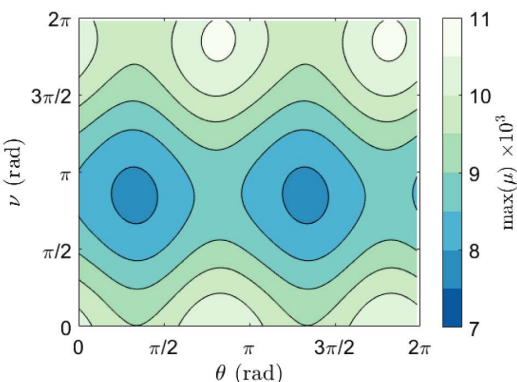

a) QSO-H

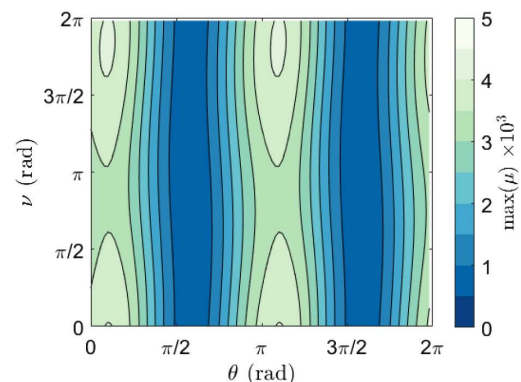

b) QSO-M

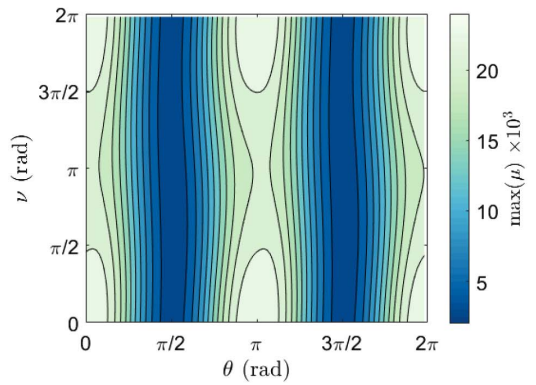

c) QSO-La

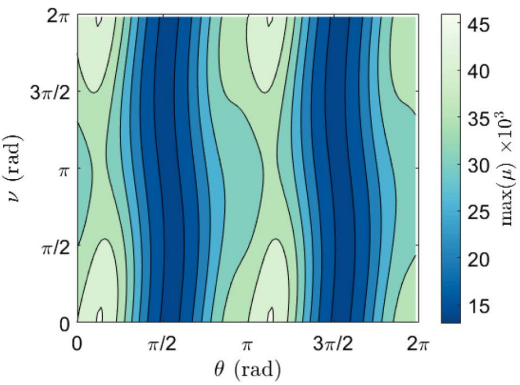

d) QSO-Lb

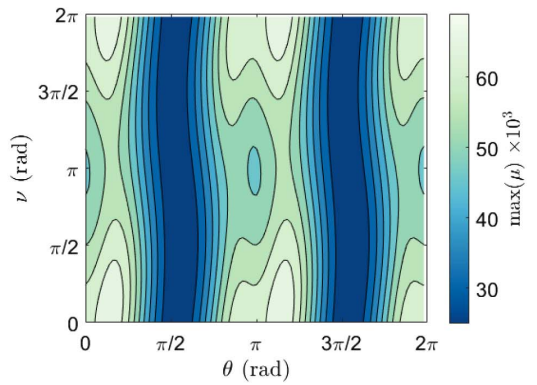

e) QSO-Lc

Fig. 5 Maximum eigenvalues of the Cauchy-Green tensor for the candidate QSOs.

matrix of a single reference trajectory; 2) unnecessary maneuvers would be triggered even though the spacecraft is actually close to other quasi-periodic trajectories that belong to the same invariant torus, although at a different longitude.

Following the discussion of Sec. IV, we propose to update the reference trajectory based on the estimated value of the spacecraft longitude, evaluate if a maneuver is needed, and potentially steer MMX back to the surface of the desired torus with a guidance of choice, e.g., an LQR controller. We pick the spacecraft longitude as the major driver for the generation of new reference trajectories because there is only one possible value of $\lambda$ for any given value of $\nu$ (imagine drawing a line parallel to the $x$ axis in the central figures of Figs. 2 and 3 ). Thus, if $\hat{\boldsymbol{X}}$ is the estimated state of MMX when Phobos's true anomaly is equal to $\hat{\nu}$, we can calculate the estimated longitude of the spacecraft, namely, $\hat{\lambda}$, and invert the maps of Figs. $\underline{2 b}$ and $2 \mathrm{e}$ and of Figs. $3 \mathrm{~b}, 3 \mathrm{e}$, and $3 \mathrm{~h}$ to find the corresponding value of $\theta(\hat{\nu}, \bar{\lambda})=\hat{\theta}$. Given $(\hat{\theta}, \hat{\nu})$, we can generate a new reference trajectory from the trivial dynamics of Eq. (26) and quickly obtain a new time history of phase space coordinates via $2 \mathrm{D}$ interpolation of state coordinates over $(\hat{\theta}+2 \pi(\nu-\hat{\nu}) / T, \nu)$. A sample update of reference trajectory is shown in Fig. 6 . This process is repeated at each

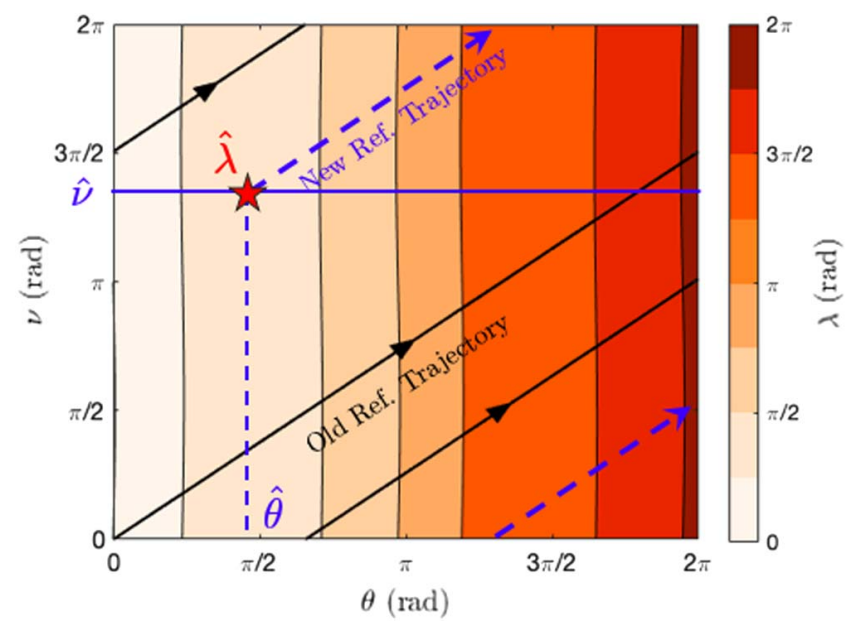

Fig. 6 Reference trajectory update process via 2D torus maps. navigation epoch to assess whether the relative distance between $\hat{X}$ and the newly found quasi-periodic trajectory is large enough to prompt a maneuver. In the case where maneuver becomes necessary, the estimated state of the spacecraft is propagated until the beginning of the thrusting arc (by design, $1 \mathrm{~h}$ after the navigation cutoff) and used to generate a new reference trajectory that shall be approached in less than $6 \mathrm{~h}$ (this number was based on preliminary navigation analyses made in JAXA), i.e., the available time of a typical observation window. During this time frame, optimal control policies that would nullify the relative error between the estimated state of the spacecraft and its desired location are computed and uploaded in an open-loop fashion to the satellite.

Specifically, if

$$
\dot{\hat{\boldsymbol{X}}}=\boldsymbol{f}\left(\nu, \hat{\boldsymbol{X}}, e_{P}\right)+B \boldsymbol{u}
$$

stands for the equations of motion of the controlled estimated trajectory, with $B=\left[0_{3 \times 3}, I_{3 \times 3}\right]^{\mathrm{T}}$, and $\boldsymbol{X}^{*}$ as the newly found reference trajectory, and then the error vector is defined as

$$
\begin{aligned}
\dot{\boldsymbol{e}} & =\dot{\hat{\boldsymbol{X}}}-\dot{\boldsymbol{X}}^{*} \\
& =\boldsymbol{f}\left(\nu, \hat{\boldsymbol{X}}, e_{P}\right)+B \boldsymbol{u}-\boldsymbol{f}\left(\nu, \boldsymbol{X}^{*}, e_{P}\right), \\
& \simeq A\left(\nu, \boldsymbol{X}^{*}, e_{P}\right) \boldsymbol{e}+B \boldsymbol{u}
\end{aligned}
$$

Note that $A=\nabla \boldsymbol{f}\left(\nu, \boldsymbol{X}^{*}, e_{P}\right)$ is the Jacobian of the vector field in Eq. (8) evaluated along $\boldsymbol{X}^{*}$. To nullify the error vector $\boldsymbol{e}$, the cost function

$$
J(\boldsymbol{e}, \boldsymbol{u})=\int_{\nu}^{(\nu+\delta \nu)}\left(\boldsymbol{e}^{\mathrm{T}} P \boldsymbol{e}+\boldsymbol{u}^{\mathrm{T}} R \boldsymbol{u}\right) \mathrm{d} \nu
$$

is minimized by the optimal control policy given by [57]

$$
\boldsymbol{u}=-R^{-1} B K \boldsymbol{e}
$$

where $P$ and $R$ are user-defined weighing matrices, and $K$ is the timevarying solution of the differential Riccati equation:

$$
\dot{K}=(K B) R^{-1}\left(B^{\mathrm{T}} K\right)-K A-A^{\mathrm{T}} K-P
$$


Table 3 MMX injection errors

\begin{tabular}{lcc}
\hline \hline Error & Value & Unit \\
\hline $1 \sigma$ Position & {$[50,50,50]$} & $\mathrm{m}$ \\
$\sigma$ Velocity & {$[3,3,3]$} & $\mathrm{cm} / \mathrm{s}$ \\
\hline \hline
\end{tabular}

The orbit maintenance analysis for the candidate quasi-satellite orbits is carried out under the assumption that MMX will be inserted on a quasi-satellite orbit with zero-mean Gaussian distribution errors with standard deviations as in Table 3 . These errors are provided by the system design team of MMX based on preliminary orbit determination performances. The insertion point is located along the negative $y$ axis crossing of a quasi-satellite orbit when $\nu=0$ deg. Furthermore, the state of the spacecraft is determined once a day within zero-mean Gaussian distribution errors similar to the ones of Table 3. Throughout the orbital propagations, a maneuver is triggered if the relative error between $\hat{\boldsymbol{X}}$ and $\boldsymbol{X}^{*}$ at the navigation cutoff time is greater than $5 \%$ of the nominal altitude for the lower altitude QSOs, and greater than $10 \%$ of the nominal altitude for QSO-H and QSO-M. Here, the more relaxed constraint for QSO-H and QSO-M is motivated by their lower sensitivity to initial errors of MMX as shown in Sec. V. If implemented, a maneuver should start $1 \mathrm{~h}$ after orbit determination and last for no longer than $6 \mathrm{~h}$. This worst-case scenario simulates communication delays and maximum observation windows (approximately $8 \mathrm{~h}$ ) with a $1 \mathrm{~h}$ margin. A $3 \sigma 5 \%$ Gaussian magnitude execution error is added to the nominal control profiles during maneuvers, along with a $3 \sigma 0.1$ deg zero-mean Gaussian error on the azimuth and elevation of the thrusting direction. These preliminary values were also provided by the system dynamics team of MMX, noting that the system design is currently under development, and that low-thrust technologies are currently not being considered. That is, the results of our continuous thrust simulations will be part of future $\Delta V$-budget and tradeoff analyses.

For each of the candidate QSOs, we have performed a Monte Carlo simulation of 1000 samples and recorded the total number of maneuvers as well as the total $\Delta V$ cost required for maintaining MMX around its nominal altitude for more than a month. The weighing matrices were initialized as $P=\operatorname{diag}\left(\left[1,1,10^{-6}, 1,1,10^{-6}\right]\right)$ and $R=10^{-4} I_{3 \times 3}$ to put emphasis on the in-plane components and penalize the fuel cost in the minimization process. Based on these values, the differential Riccati equation in Eq. (32) was integrated backward for approximately $12 \mathrm{~h}$ starting from the initial condition $K=0_{6 \times 6}$. Only the last $6 \mathrm{~h}$ of this integration was stored and passed to the LQR controller so as to avoid transient effects and meet the operational requirements set up in our assumptions.

The output of the orbit maintenance analysis is shown in Fig. 7. The cumulative distribution function for the total orbit maintenance cost and the total number of required thrusting arcs are shown in Figs. 7a and 7b, respectively. It appears that maneuvers may not be necessary for the majority of cases. In fact, QSO-M and QSO-H do not require any orbit maintenance maneuvers as long as the tolerance of their relative error with respect to their nominal altitude profile is set to $10 \%$. Conversely, QSO-La, QSO-Lb, and QSO-Lc may require a few maneuvers per month, but only in the $42 \%, 15 \%$, and $11 \%$ of the cases, respectively. This reflects the stable nature of the computed quasi-satellite orbits observed in Sec. IV. When needed, maneuvers can successfully bring MMX close to its nominal altitude with a total $\Delta V$ cost of $6.265 \mathrm{~m} / \mathrm{s}$ at worst. This value has been recorded for the QSO-La orbit, and it is larger than the maximum value of the total $\Delta V$ cost observed for the QSO-Lb and QSO-Lc cases: 3.779 and $2.577 \mathrm{~m} / \mathrm{s}$, respectively. We suspect that this difference may be due to the narrower stability islands found in the vicinity of the QSO-La case and reported in Ref. [46]. A sample control profile and controlled trajectory are disclosed in Fig. 8 for the case of QSO-La. The current maximum value of $6.265 \mathrm{~m} / \mathrm{s}$ per month is well within the current $\Delta V$ budget capabilities of MMX and it is considered as a low expenditure.

\section{Conclusions}

This paper presented the latest improvements on the trajectory design and orbit maintenance analyses for the Martian Moon eXploration mission around Phobos. Starting from the equations of the elliptic Hill problem with a constant density ellipsoidal model, this paper introduced a numerical continuation procedure to calculate families of quasi-periodic orbits for increasing values of Phobos eccentricity. The numerical continuation scheme was stopped at $e_{P}=$ 0.0151 (our current best estimate) and used to produce the dynamic substitutes of five quasi-satellite orbits being considered for the MMX mission.

It was verified that these solutions are stable even when the eccentricity of Phobos is taken into account and observed that the points of minimum sensitivity seem to be located above the leading and trailing edges of the Phobes. Based on this information, MMX was injected in the along-track direction of Phobos and a Monte Carlo simulation was run to evaluate the impact of injection and navigation errors on the relative dynamics. Reference trajectories are updated and generated using a quick and simple methodology enabled by 2D torus maps. By design, the updated reference trajectories are close to the actual trajectory of MMX, so that classic linear methods may succeed in preserving the altitude of the spacecraft within operational requirements. The results of our Monte Carlo analysis revealed that our station-keeping approach maintains all of the considered QSOs with $6.265 \mathrm{~m} / \mathrm{s}$ per month at worst. It has also been shown that higher altitude QSOs do not necessitate orbit maintenance maneuvers altogether. QSO-H and QSO-M may thus be used as safe areas for MMX operations and/or contingencies plans.

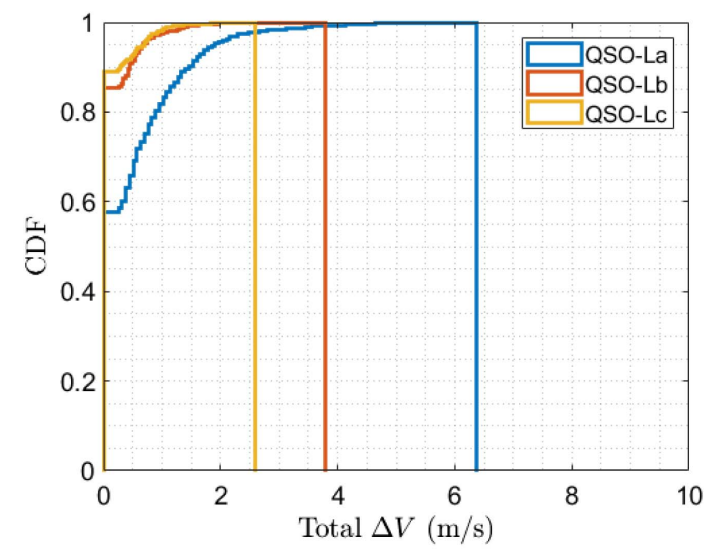

a) CDFs of the total $\Delta V$ cost over one month

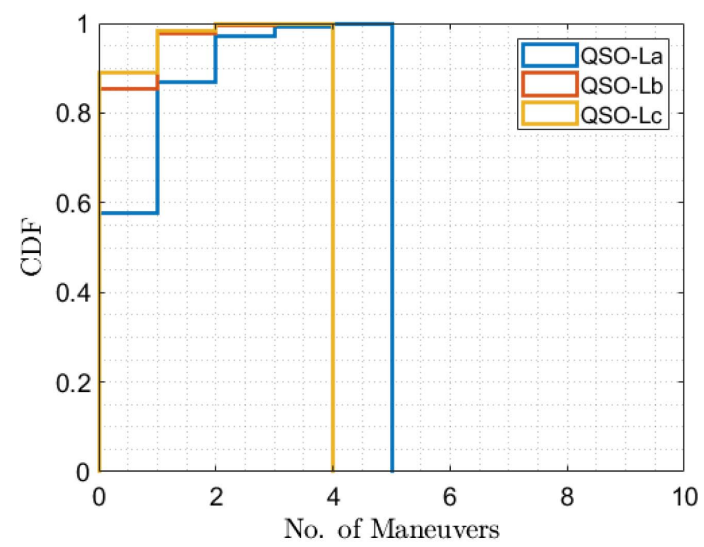

b) CDFs of the no. of maneuvers over one month

Fig. 7 Cumulative distribution functions of total $\Delta V$ cost and number of maneuvers for 1000 Monte Carlo samples near lower-altitude QSOs. No maneuvers were found for QSO-M and QSO-H. 


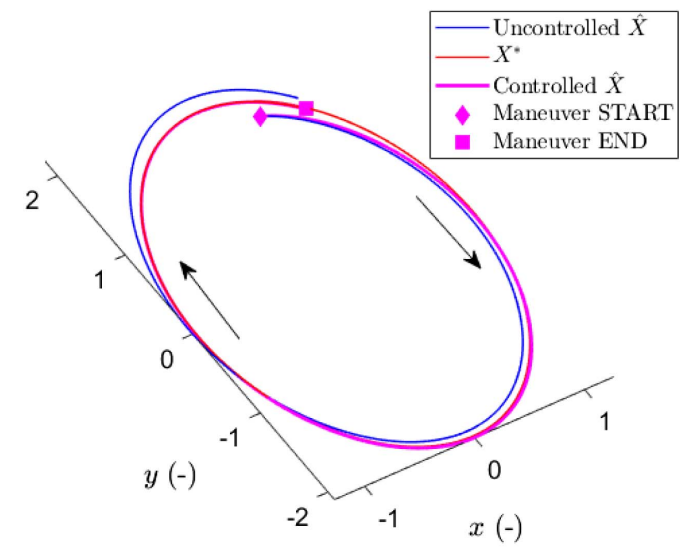

a) Uncontrolled, controlled, and reference LQR trajectories in pulsating synodic coordinates

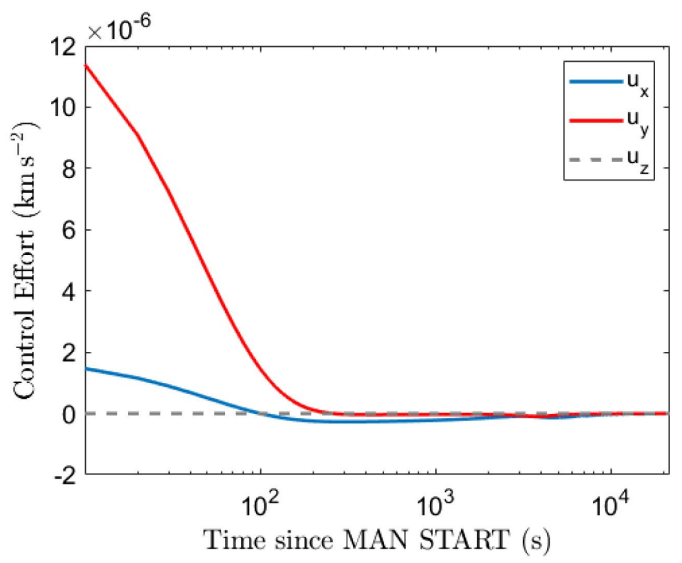

b) Control effort over 6 hours

Fig. 8 Sample LQR maneuver triggered during one of the QSO-La Monte Carlo samples.

\section{Appendix A: Partials of Vector Field with Respect to State Vector}

To calculate $[\partial \boldsymbol{f} / \partial \boldsymbol{X}]$, i.e., the partial derivatives of Eq. (8) with respect to the pulsating coordinates $\boldsymbol{x}=[x, y, z, \dot{x}, \dot{y}, \dot{z}]^{\mathrm{T}}$, recall that the gravitational acceleration due to an ellipsoidal Phobos, $\boldsymbol{G}$, is a function of the dimensional components of the spacecraft position vector as seen from the principal axis frame of the Martian moon: $\boldsymbol{R}_{b}=[X, Y, Z]^{\mathrm{T}}=\epsilon p_{P} \gamma^{-1} R_{z}[-\Upsilon] \boldsymbol{r}$. Accordingly,

$$
\begin{aligned}
{\left[\frac{\partial \boldsymbol{g}}{\partial \boldsymbol{x}}\right] } & =\kappa R_{z}[\Upsilon]\left[\frac{\partial \boldsymbol{G}}{\partial \boldsymbol{x}}\right] \\
& =\kappa R_{z}[\Upsilon]\left[\frac{\partial \boldsymbol{G}}{\partial \boldsymbol{R}_{b}}\right]\left[\frac{\partial \boldsymbol{R}_{b}}{\partial \boldsymbol{x}}\right] \\
& =\mu_{M}^{-1} p_{P}^{3} \gamma^{-4} R_{z}[\Upsilon]\left[\frac{\partial \boldsymbol{G}}{\partial \boldsymbol{R}_{b}}\right] R_{z}[-\Upsilon]
\end{aligned}
$$

where

$$
\begin{gathered}
{\left[\frac{\partial \boldsymbol{G}}{\partial \boldsymbol{R}_{b}}\right]=\left[\begin{array}{lll}
G_{x x}, & G_{x y}, & G_{x z}, \\
G_{x y}, & G_{y y}, & G_{y z}, \\
G_{x z}, & G_{y z}, & G_{z z}
\end{array}\right]} \\
G_{x x}=\frac{3 \mu_{P} X^{2}}{\left(\bar{\alpha}^{2}+\Lambda\right)^{2} \Delta(\Lambda)} \frac{1}{\Psi(X, Y, Z, \Lambda)} \\
-\frac{3 \mu_{P}}{2} \int_{0}^{\infty}\left(\frac{1}{\bar{\alpha}^{2}+\Lambda+l}\right) \frac{\mathrm{d} l}{\Delta(l+\Lambda)} \\
G_{y y}=\frac{3 \mu_{P} Y^{2}}{\left(\bar{\beta}^{2}+\Lambda\right)^{2} \Delta(\Lambda)} \frac{1}{\Psi(X, Y, Z, \Lambda)} \\
-\frac{3 \mu_{P}}{2} \int_{0}^{\infty}\left(\overline{\bar{\beta}^{2}+\Lambda+l}\right) \frac{1}{\Delta(l+\Lambda)} \\
G_{z z}=\frac{3 \mu_{P} Z^{2}}{\left(\bar{\alpha}^{2}+\Lambda\right)^{2} \Delta(\Lambda)} \frac{1}{\Psi(X, Y, Z, \Lambda)} \\
-\frac{3 \mu_{P}}{2} \int_{0}^{\infty}\left(\frac{1}{\bar{\gamma}^{2}+\Lambda+l}\right) \frac{\mathrm{d} l}{\Delta(l+\Lambda)} \\
G_{x y}=\frac{3 \mu_{P} X Y}{\left(\bar{\alpha}^{2}+\Lambda\right)^{2}\left(\bar{\beta}^{2}+\Lambda\right)^{2} \Delta(\Lambda)} \frac{1}{\Psi(X, Y, Z, \Lambda)} \\
+\Lambda \mu^{2}\left(\bar{\gamma}^{2}+\Lambda\right)^{2} \Delta(\Lambda) \frac{1}{\Psi(X, Y, Z, \Lambda)}
\end{gathered}
$$

$$
\begin{gathered}
G_{y z}=\frac{3 \mu_{P} Y Z}{\left(\bar{\beta}^{2}+\Lambda\right)^{2}\left(\bar{\gamma}^{2}+\Lambda\right)^{2} \Delta(\Lambda)} \frac{1}{\Psi(X, Y, Z, \Lambda)} \\
\Psi(X, Y, Z, \Lambda)=\left[\frac{X^{2}}{\left(\bar{\alpha}^{2}+\Lambda\right)^{2}}+\frac{Y^{2}}{\left(\bar{\beta}^{2}+\Lambda\right)^{2}}+\frac{Z^{2}}{\left(\bar{\gamma}^{2}+\Lambda\right)^{2}}\right]
\end{gathered}
$$

Then,

$$
\left[\frac{\partial \boldsymbol{f}}{\partial \boldsymbol{X}}\right]=\left[\begin{array}{cc}
0_{3 \times 3}, & I_{3 \times 3} \\
{\left[\frac{\partial \boldsymbol{g}}{\partial \boldsymbol{x}}\right]+3 \frac{\hat{\boldsymbol{x}} \hat{\boldsymbol{x}}^{\mathrm{T}}}{\gamma}-\hat{z} \hat{\boldsymbol{z}}^{\mathrm{T}},} & -2[\hat{\boldsymbol{z}}]
\end{array}\right]
$$

where $0_{3 \times 3}$ is the null matrix, $I_{3 \times 3}$ is the identity matrix, and

$$
[\hat{z}]=\left[\begin{array}{ccc}
0, & -1, & 0 \\
1, & 0, & 0 \\
0, & 0, & 0
\end{array}\right]
$$

\section{Appendix B: Partials of Vector Field with Respect to Eccentricity}

As for $[\partial f / \partial e]$, i.e., the partial derivatives of Eq. ( $\underline{8})$ with respect to $e$, observe that the eccentricity of the Phobos appears explicitly in the definitions of

$$
\begin{aligned}
p_{P} & =a_{P}\left(1-e_{P}^{2}\right) \\
\gamma & =1+e_{P} \cos \nu \\
\theta & =v-E+e_{P} \sin E \\
E & =2 \arctan \left[\sqrt{\frac{1-e_{P}}{1+e_{P}}} \tan \left(\frac{\nu}{2}\right)\right]
\end{aligned}
$$

Accordingly,

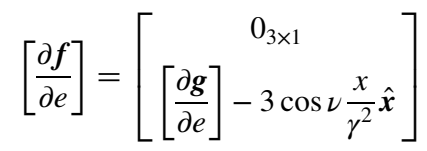

where

$$
\begin{aligned}
{\left[\frac{\partial \boldsymbol{g}}{\partial e}\right] } & =\kappa_{e} R_{z}[\Upsilon] \boldsymbol{G}+\kappa \frac{\partial}{\partial e}\left(R_{z}[\Upsilon]\right) \boldsymbol{G}+\kappa R_{z}[\Upsilon]\left[\frac{\partial \boldsymbol{G}}{\partial e}\right] \\
\kappa_{e} & =-\kappa\left[4 e \gamma+3 \cos \nu\left(1-e^{2}\right)\right]\left(1-e^{2}\right)^{-1} \gamma^{-1}
\end{aligned}
$$




$$
\begin{gathered}
\frac{\partial}{\partial e}\left(R_{z}[\Upsilon]\right)=\Upsilon_{e}\left[\begin{array}{ccc}
-\sin \Upsilon, & \cos \Upsilon, & 0 \\
-\cos \Upsilon, & -\sin \Upsilon, & 0 \\
0, & 0, & 0
\end{array}\right] \\
\Upsilon_{e}=\sin E-(1-e \cos E) E_{e} \\
E_{e}=-\sin \nu\left(1-e^{2}\right)^{-1 / 2} \gamma^{-1} \\
{\left[\frac{\partial \boldsymbol{G}}{\partial e}\right]=\left[\frac{\partial \boldsymbol{G}}{\partial \boldsymbol{R}_{b}}\right]\left[\frac{\partial \boldsymbol{R}_{b}}{\partial e}\right]} \\
{\left[\frac{\partial \boldsymbol{R}_{b}}{\partial e}\right]=\varepsilon p \gamma^{-1} \boldsymbol{r}\left[-2 e\left(1-e^{2}\right)^{-1} R_{z}[-\Upsilon]\right.} \\
\left.-\cos \nu \gamma^{-1} R_{z}[-\Upsilon]+\frac{\partial}{\partial e}\left(R_{z}[\Upsilon]\right)^{\mathrm{T}}\right]
\end{gathered}
$$

\section{Acknowledgments}

13 This work has been partially supported by JSPS Grants-in-Aid No. 19F18371. The authors would like to acknowledge F. GonzalezFranquesa, N. Pushparaj, and T. Chikazawa for their valuable inputs in the literature review of the exploration of the Martian moons.

\section{References}

[1] Burns, J. A., "Dynamical Characteristics of Phobos and Deimos," Reviews of Geophysics, Vol. 10, No. 2, 1972, pp. 463-483. https://doi.org/10.1029/RG010i002p00463

[2] Rosenblatt, P., "The Origin of the Martian Moons Revisited," Astronomy and Astrophysics Review, Vol. 19, No. 1, 2011, p. 44. https://doi.org/10.1007/s00159-011-0044-6

[3] Ballouz, R.-L., Baresi, N., Crites, S. T., Kawakatsu, Y., and Fujimoto, M., "Surface Refreshing of Martian Moon Phobos by Orbital Eccentricity-Driven Grain Motion," Nature Geoscience, Vol. 12, No. 4, 2019, pp. 229-234. https://doi.org/10.1038/s41561-019-0323-9

[4] Citron, R. I., Genda, H., and Ida, S., "Formation of Phobos and Deimos via a Giant Impact," Icarus, Vol. 252, May 2015, pp. 334-338. https://doi.org/10.1016/j.icarus.2015.02.011

[5] Pajola, M., Lazzarin, M., Dalle Ore, C. M., Cruikshank, D. P., Roush, T. L., Magrin, S., Bertini, I., La Forgia, F., and Barbieri, C., "Phobos as a D-Type Captured Asteroid, Spectral Modeling from 0.25 to 4.0 m," Astrophysical Journal, Vol. 777, No. 2, 2013, p. 127. https://doi.org/10.1088/0004-637X/777/2/127

[6] Duxbury, T. C., Zakharov, A. V., Hoffmann, H., and Guinness, E. A., "Spacecraft Exploration of Phobos and Deimos," Planetary and Space Science, Vol. 102, Nov. 2014, pp. 9-17. https://doi.org/10.1016/j.pss.2013.12.008

[7] Zakharov, A. V., Eismont, N. A., Gotlib, V. M., Smirnov, V. M., Yushkova, O. V., and Marchuk, V. N., "Radiosounding in the Planned Mission to Phobos," Solar System Research, Vol. 51, No. 5, 2017, pp. 386-399. https://doi.org/10.1134/S0038094617050100

[8] Pickering, A., "CDF Study Report: Phobos Sample Return," European Space Agency (ESA) Tech. Rept. CDF-145(C), 2014, https://sci.esa.int/ documents/34923/36148/1567260187106-PhobosSR_v2_Releasable.pdf.

[9] Oberst, J., Wickhusen, K., Willner, K., Gwinner, K., Spiridonova, S., Kahle, R., Coates, A., Herique, A., Plettemeier, D., Daz-Michelena, M., Zakharov, A., Futaana, Y., Pätzold, M., Rosenblatt, P., Lawrence, D. J., Lainey, V., Gibbings, A., and Gerth, I., "DePhine-The Deimos and Phobos Interior Explorer," Advances in Space Research, Vol. 62, No. 8, 2018, pp. 2220-2238. https://doi.org/10.1016/j.asr.2017.12.028

[10] Lee, P., Bicay, M., Colaprete, A., Elphic, R., Genova, A., Hine, B., Horanyi, M., Jaroux, B., Korsmeyer, D., Lewis, B. S., and Worden, S. P., "Phobos and Deimos and Mars Environment (PADME): A LADEEDerived Mission to Explore Mars's Moons and the Martian Orbital Environment," 45th Lunar and Planetary Science Conference, Lunar and Planetary Inst., Houston, TX, 2014, Abstract \#2288, http://www.lpi .usra.edu/meetings/lpsc2014/pdf/2288.pdf.

[11] Murchie, S. L., Chabot, N. L., Buczkowski, D. L., Eng, D. A., Peplowski, P. N., Ernst, C. M., Seelos, F. P., Horanyi, M., Castillo-Rogez, J. C., Chmielewski, A. B., Maki, J. N., Trebi-Ollenu, A., Ehlmann, B. L., Klingelhoefer, G., Arvidson, R. E., Spence, H. E., and Christian, J. A. A.,
"Mars-Moons Exploration, Reconnaissance, and Landed Investigation (MERLIN)," 2016 IEEE Aerospace Conference, Inst. of Electrical and Electronics Engineers, New York, 2016, pp. 1-18. https://doi.org/10.1109/AERO.2016.7500754

[12] Raymond, C. A., Prettyman, T. H., and DIniega, S., "PANDORAUnlocking the Mysteries of the Moons of Mars," 46th Lunar and Planetary Science Conference, Lunar and Planetary Inst., Houston, TX, 2015, Abstract \#2792, http://www.lpi.usra.edu/meetings/lpsc2015/ pdf/2792.pdf.

[13] Arya, A. S., Moorthi, S. M., Rajasekhar, R. P., Sarkar, S. S., Sur, K., Aravind, B., Gambhir, R. K., Misra, I., Patel, V. D., Srinivas, A. R., Patel, K. K., Chauhan, P., and Kiran Kumar, A. S., "Indian Mars-Colour-Camera Captures Far-Side of the Deimos: A Rarity Among Contemporary Mars Orbiters," Planetary and Space Science, Vol. 117, Nov. 2015, pp. 470474. https://doi.org/10.1016/j.pss.2015.08.018

[14] Witasse, O., Duxbury, T., Chicarro, A., Altobelli, N., Andert, T., Aronica, A., Barabash, S., Bertaux, J.-L., Bibring, J.-P., Cardesin-Moinelo, A., Cichetti, A., Companys, V., Dehant, V., Denis, M., Formisano, V., Futaana, Y., Giuranna, M., Gondet, B., Heather, D., Hoffmann, H., Holmström, M., Manaud, N., Martin, P., Matz, K.-D., Montmessin, F., Morley, T., Mueller, M., Neukum, G., Oberst, J., Orosei, R., Pätzold, M., Picardi, G., Pischel, R., Plaut, J., Reberac, A., Voss, P. P., Roatsch, T., Rosenblatt, P., Remus, S., Schmedemann, N., Willner, K., and Zegers, T., "Mars Express Investigations of Phobos and Deimos," Planetary and Space Science, Vol. 102, Nov. 2014, pp. 18-34. https://doi.org/10.1016/j.pss.2013.08.002

[15] Kawakatsu, Y., Kuramoto, K., Usui, T., Ikeda, H., Ozaki, N., Baresi, N., Ono, G., Imada, T., Shimada, T., Kusano, H., Sawada, H., Ozawa, T., Baba, M., and Otake, H., "Mission Design of Martian Moons eXploration (MMX)," 69th International Astronautical Congress (IAC), 2018, pp. $1-12$.

[16] Ikeda, H., Mitani, S., Mimasu, Y., Ono, G., Nigo, K., and Kawakatsu, Y., "Orbital Operations Strategy in the Vicinity of Phobos," 25th International Symposium on Space Flight Dynamics (ISTS), 2017, pp. 1-8.

[17] Darwin, G. H., "Periodic Orbits," Acta Mathematica, Vol. 21, No. 1, 1897, pp. 99-242. https://doi.org/10.1007/BF02417978

[18] Jackson, J., "Retrograde Satellite Orbits," Monthly Notices of the Royal Astronomical Society, Vol. 74, Dec. 1913, pp. 62-82. https://doi.org/10.1093/mnras/74.2.62.

[19] Hénon, M., "Numerical Exploration of the Restricted Problem, V," Astronomy and Astrophysics, Vol. 1, Feb. 1969, pp. 223-238.

[20] Marov, M. Y., Avduevsky, V. S., Akim, E. L., Eneev, T. M., Kremnev, R. S., Kulikov, S. D., Pichkhadze, K. M., Popov, G. A., and Rogovsky, G. N., "Phobos-Grunt: Russian Sample Return Mission," Advances in Space Research, Vol. 33, No. 12, 2004, pp. 2276-2280. https://doi.org/10.1016/S0273-1177(03)00515-5

[21] Strange, N., Landau, D., McElrath, T., Lantoine, G., Lam, T., McGuire, M., Burke, L., Martini, M., and Dankanich, J., "Overview of Mission Design for NASA Asteroid Redirect Robotic Mission Concept," 33rd International Electric Propulsion Conference, 2013, pp. 1-12.

[22] Canalias, E., Lorda, L., and Laurent-Varin, J., "Design of Realistic Trajectories for the Exploration of Phobos," 2018 Space Flight Mechanics Meeting, AIAA Paper 2018-0716, 2018, pp. 1-15. https://doi.org/10.2514/6.2018-0716

[23] Lidov, M. L., and Vashkov'yak, M. A., "Perturbation Theory and Analysis of the Evolution of Quasi-Satellite Orbits in the Restricted ThreeBody Problem," Cosmic Research, Vol. 31, No. 2, 1993, pp. 187-207.

[24] Gil, P. J. S., and Schwartz, J., "Simulations of Quasi-Satellite Orbits Around Phobos," Journal of Guidance, Control, and Dynamics, Vol. 33 , No. 3, 2010, pp. 901-914. https://doi.org/10.2514/1.44434

[25] Zamaro, M., and Biggs, J. D., "Natural Motion Around the Martian Moon Phobos: The Dynamical Substitutes of the Libration Point Orbits in an Elliptic Three-Body Problem with Gravity Harmonics," Celestial Mechanics and Dynamical Astronomy, Vol. 122, No. 3, 2015, pp. 263-302. https://doi.org/10.1007/s10569-015-9619-2

[26] Wiesel, W. E., "Stable Orbits About the Martian Moons," Journal of Guidance, Control, and Dynamics, Vol. 16, No. 3, 1993, pp. 434-440. https://doi.org/10.2514/3.21028

[27] Scheeres, D. J., Van Wal, S., Olikara, Z., and Baresi, N., "Dynamics in the Phobos Environment," Advances in Space Research, Vol. 63, No. 1, 2019, pp. 476-495. https://doi.org/10.1016/j.asr.2018.10.016

[28] Shirobokov, M., Trofimov, S., and Ovchinnikov, M., "Survey of StationKeeping Techniques for Libration Point Orbits," Journal of Guidance, Control, and Dynamics, Vol. 40, No. 5, 2017, pp. 1085-1105. https://doi.org/10.2514/1.G001850 
29] Guzzetti, D., Zimovan, E. M., Howell, K. C., and Davis, D. C., "Station Keeping Analysis for Spacecraft in Lunar Near Rectilinear Halo Orbits," 27th AAS/AIAA Space Flight Mechanics Meeting, 2017, Paper AAS-395.

[30] Simó, C., Gómez, J., Llibre, G., Martinez, R., and Rodriguez, J., "On the Optimal Station Keeping Control of Halo Orbits," Acta Astronautica, Vol. 15, Nos. 6-7, 1987, pp. 391-397. https://doi.org/10.1016/0094-5765(87)90175-5

[31] Scheeres, D. J., Hsiao, F.-Y., and Vinh, N. X., "Stabilizing Motion Relative to an Unstable Orbit: Applications to Spacecraft Formation Flight," Journal of Guidance, Control, and Dynamics, Vol. 26, No. 1, 2003, pp. 62-73. https://doi.org/10.2514/2.5015

[32] Soldini, S., Colombo, C., and Walker, S. J., "Comparison of Hamiltonian Structure-Preserving and Floquét Mode Station-Keeping for LibrationPoint Orbits," 2014 AIAA/AAS Astrodynamics Specialist Conference, AIAA Paper 2014-4118, 2014, pp. 1-21. https://doi.org/10.2514/6.2014-4118

[33] Folta, D. C., Pavlak, T. A., Haapala, A. F., Howell, K. C., and Woodard, M. A., "Earth-Moon Libration Point Orbit Stationkeeping: Theory, Modeling, and Operations," Acta Astronautica, Vol. 94, No. 1, 2014, pp. 421-433. https://doi.org/10.1016/j.actaastro.2013.01.022

[34] Howell, K. C., and Pernicka, H. J., "Stationkeeping Method for Libration Point Trajectories," Journal of Guidance, Control, and Dynamics, Vol. 16, No. 1, 1993, pp. 151-159.

https://doi.org/10.2514/3.11440

[35] Howell, K. C., and Gordon, S. C., "Orbit Determination Error Analysis and a Station-Keeping Strategy for Sun-Earth L1 Libration Point Orbits," Journal of the Astronautical Sciences, Vol. 42, No. 2, 1994, pp. 207-228.

[36] Cipriano, A. M., Dei Tos, D. A., and Topputo, F., “Orbit Design for LUMIO: The Lunar Meteoroid Impacts Observer," Frontiers in Astronomy and Space Sciences, Vol. 5, Sept. 2018, p. 29. https://doi.org/10.3389/fspas.2018.00029

[37] Oguri, K., Oshima, K., Campagnola, S., Kakihara, K., Ozaki, N., Baresi, N., Kawakatsu, Y., and Funase, R., "EQUULEUS Trajectory Design," Journal of the Astronautical Sciences, Vol. 67, Jan. 2020, pp. 950-976. https://doi.org/10.1007/s40295-019-00206-y

[38] Cielaszyk, D., and Wie, B., "New Approach to Halo Orbit Determination and Control," Journal of Guidance, Control, and Dynamics, Vol. 19, No. 2, 1996, pp. 266-273. https://doi.org/10.2514/3.21614

[39] Guo, Y., Hawkins, M., and Wie, B., "Applications of Generalized ZeroEffort-Miss/Zero-Effort-Velocity Feedback Guidance Algorithm,' Journal of Guidance, Control, and Dynamics, Vol. 36, No. 3, 2013, pp. 810-820. https://doi.org/10.2514/1.58099

[40] Wibben, D. R., and Furfaro, R., "Optimal Sliding Guidance Algorithm for Mars Powered Descent Phase," Advances in Space Research, Vol. 57, No. 4, 2016, pp. 948-961. https://doi.org/10.1016/j.asr.2015.12.006

[41] Drozd, K. M., Furfaro, R., and Topputo, F., "Application of ZEM/ZEV Guidance for Closed-Loop Transfer in the Earth-Moon System," 2018 Space Flight Mechanics Meeting, AIAA Paper 2018-0958, 2018. https://doi.org/10.2514/6.2018-0958

[42] Scorsoglio, A., Furfaro, R., Linares, R., and Massari, M., "Actor-Critic Reinforcement Learning Approach to Relative Motion Guidance in Near-Rectilinear Orbit," 29th AAS/AIAA Space Flight Mechanics Meeting, 2019, pp. 1-20.
[43] Elliott, I., Bosanac, N., Ahmed, N. R., and McMahon, J. W., "Apprenticeship Learning for Maneuver Design in Multi-Body Systems," AIAA SciTech 2020 Forum, AIAA Paper 2020-1912, 2020, pp. 117.

https://doi.org/10.2514/6.2020-1912

[44] Guzzetti, D., Sood, R., Chappaz, L., and Baoyin, H., "Stationkeeping Analysis for Solar Sailing the L4 Region of Binary Asteroid Systems," Journal of Guidance, Control, and Dynamics, Vol. 42, No. 6, 2019, pp. 1306-1318. https://doi.org/10.2514/1.G003994

[45] Akiyama, Y., Bando, M., and Hokamoto, S., "Explicit Form of StationKeeping and Formation Flying Controller for Libration Point Orbits," Journal of Guidance, Control, and Dynamics, Vol. 41, No. 6, 2018, pp. 1407-1415. https://doi.org/10.2514/1.G002845

[46] Baresi, N., and Kawakatsu, Y., "Quasi-Periodic Motion Around Phobos: Applications to the Martian Moons eXploration (MMX)," 32nd International Symposium on Space Technology and Science (ISTS), 2019, pp. 1-8.

[47] Murchie, S. L., Thomas, P. C., Rivkin, A. S., and Chabot, N. L., "Phobos and Deimos," Asteroids IV, edited by P. Michel, F. E. Demeo, and W. F. Bottke, Univ. of Arizona Press, Tucson, AZ, 2015, pp. 451-467. https://doi.org/10.2458/azu_uapress_9780816532131-ch024

[48] Scheeres, D. J., "Dynamics About Uniformly Rotating Triaxial Ellipsoids: Applications to Asteroids," Icarus, Vol. 110, No. 2, 1994, pp. 225 238.

https://doi.org/10.1006/icar.1994.1118

[49] Press, W. H., Teukolsky, S. A., Vetterling, W. T., and Flannery, B. P., Numerical Recipes, 3rd ed., The Art of Scientific Computing, Cambridge Univ. Press, Cambridge, MA, 2007.

[50] Baresi, N., Olikara, Z. P., and Scheeres, D. J., " $\mathrm{F}]$ " umerical Methods for Continuing Families of Quasi-Periodic Invariant Tori in Astrodynamics," Journal of the Astronautical Sciences, Vol. 65, No. 2, 2018, pp. 157-182. https://doi.org/10.1007/s40295-017-0124-6

[51] Olikara, Z. P., "Computation of Quasi-Periodic Tori and Heteroclinic Connections in Astrodynamics Using Collocation Techniques," Ph.D. Thesis, Univ. of Colorado, Boulder, CO, 2016, https://scholar.colorado .edu/asen_gradetds/144/.

[52] Seydel, R., Practical Bifurcation and Stability Analysis, Vol. 5, Springer Science \& Business Media, 2009.

[53] Jorba, À., "Numerical Computatio e Normal Behaviour of Invariant Curves of n-Dimensional Maps," Nonlinearity, Vol. 14, No. 5, 2001, pp. 943-976. https://doi.org/10.1088/0951-7715/14/5/303

[54] Olikara,Z., and Scheeres, D., "Numerical Method for Computing QuasiPeriodic Orbits and their Stability in the Restricted Three-Body Problem," Advances in the Astronautical Sciences, Vol. 145, March 2012, pp. 911-930.

[55] Baresi, N., "Spacecraft Formation Flight on Quasi-periodic Invariant Tori," Ph.D. Thesis, Univ. of Colorado, Boulder, CO, 2017, https:// scholar.colorado.edu/asen_gradetds/176/.

[56] Short, C. R., Blazevski, D., Howell, K. C., and Haller, G., "Stretching in Phase Space and Applications in General Nonautonomous Multi-Body Problems," Celestial Mechanics and Dynamical Astronomy, Vol. 122, No. 3, 2015, pp. 213-238. https://doi.org/10.1007/s10569-015-9617-4

[57] Lawden, D. F., Analytical Methods of Optimization, Dover, Mineola, NY, 2006. 


\section{Queries}

1. AU: Please expand JSPS and MMX in affiliation footnotes.

2. AU: Please check that the copyright (C) type is correct. Please note that the code will be added upon publication.

3. AU: Abstract has been edited to be in the third person, per journal guidelines. Please check that your meaning was retained.

4. AU: The nomenclature has been reordered, edited, and formatted per the style of AIAA. Please check.

5. AU: Please expand LQR.

6. AU: Please expand LQR.

7. AU: Please define LQR.

8. AU: Please define GMOS.

9. AU: Please note that some of the inline equations were made into display equations for readability and per journal guidelines.

10. AU: The citations of figures in the text have been edited per the style of AIAA. Please check if the edits are correct.

11. AU: The footnote has been incorporated into the text per the style of AIAA. Please check.

12. AU: Conclusions section has been edited to be in the third person, per journal guidelines. Please check that your meaning was retained.

13. AU: Please expand JSPS

14. AU: For Refs. $[15,16,21,42,46]$, please provide the full name and location of the publisher (NOT the conference host).

15. AU: If Ref. [29] is a published proceeding, please provide the full name and location of the publisher (NOT of the conference host) and the page range. If it is a conference paper, please provide the paper number and the organizer's name.

16. AU: For Refs. [49,57], please provide page range or chapter number.

17. AU: For Ref. [52], please publisher location, and provide page range or chapter number.

\section{Funding Information}

The following research funding sources have been associated with your manuscript:

- Japan Society for the Promotion of Science; Award no. 19F18371; Funder ID http://dx.doi.org/10.13039/501100001691

Funding sources listed here can be 1) research grants from outside agencies or organizations or 2) if an author is employed by a U.S. government agency that directly funded this research, this employer also may be listed here as a funding agency. Please confirm that this information is complete and correct for all authors. Edit the Funding Data/Acknowledgments section of your paper if you have changes to funding agency names or grant numbers. Use the funder's full and official name. 